\title{
Parametric identification of complex modulus
}

\author{
A. Rensfelt and T. Söderström
}

June 17, 2010

\begin{abstract}
This report treats three different approaches to parametric identification of the complex modulus of a viscoelastic material. In the first approach, a nonparametric estimate is used to fit the parametric model, while in the other two the model is fitted directly from data. In all three cases, theoretical expressions for the accuracy of the estimate are derived under the assumption that the measurement noise is white and that the signal-to-noise ratio is large. The expressions are validated against both simulated and experimental data. In the case of experimental data it is seen that the theoretical expression severely underestimates the variance of the identified parameters, and one of the expressions is therefore modified to cover the case of correlated noise with much better agreement as a result.
\end{abstract}




\section{Introduction}

Viscoelastic materials, such as plexiglass and other plastics, can today be found in a wide range of practical applications. In order to make efficient use of these materials, it is of interest to understand their behavior when used in an environment where the material is subjected to dynamic load. Such dynamic load could for example be vibrations from a motor, or stress put on to the structure through collision or impact.

A viscoelastic material is characterized by its frequency dependent complex modulus $E(\omega)$, that relates uni-axial stress and strain in the material. In the frequency domain this relationship looks like

$$
\sigma(\omega)=E(\omega) \varepsilon(\omega)
$$

where $\sigma(\omega)$ and $\varepsilon(\omega)$ denotes the Fourier transformed stress and strain, respectively. Knowledge about the complex modulus is essential in understanding the materials behavior in a dynamic environment, and can be determined through different kinds of wave propagation experiments, as studied in for example $[1,4,16]$.

The complex modulus of a viscoelastic material can be identified both nonparametrically, as in $[5,8,10]$, or parametrically, as in $[11,12,16]$. In nonparametric identification, the value of the complex modulus is determined at a number of discrete frequency points $\left\{\omega_{k}\right\}$. Between these frequency points, no information is given on the complex modulus. In parametric identification on the other hand, a parametric model is fitted to the data and the information on the complex modulus is then concentrated to a small number of parameters in this model. Mechanical networks, consisting of a number of springs and dashpots (dampers) are often employed to model the complex modulus of a viscoelastic material, see for example $[6,17]$. One advantage with a parametric model is that a value of the complex modulus can be easily determined for any given frequency point. Another advantage with such an approach is that it often give estimates with an low variance.

This report treats three different approaches to finding a parametric model of the complex modulus. Two of these have been used in previous studies, see for example $[11,10]$. In the first approach, the parametric model is fitted from a nonparametric model described in, for example, [8], while in the second approach the model is fitted from the measured strain data directly. For the sake of the coming analysis these approaches are here presented in more detail and the accuracy of the estimates analyzed. The third approach is an improved version of estimating the model directly from data which has not been presented previously, and its performance is here compared to that of the other methods. In all three cases, theoretical expressions for the accuracy of the estimates are derived and validated against both simulated and experimental data.

The report is organized as follows. In the following section, the three approaches to parametric identification are described. In Section 3, theoretical expressions for the accuracy of the estimates are derived for all three approaches 


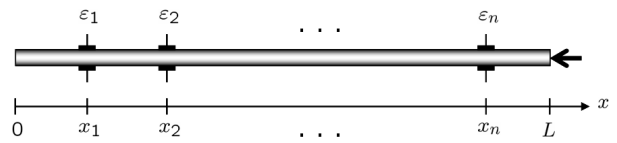

Figure 1: Experimental setup.

under the assumption that the measurement noise is white and the signal-tonoise ratio large. The accuracy expressions are also compared between two of the approaches. All expressions are validated against simulated data in Section 4 and against experimental data in Section 5. In Section 6, one of the theoretical accuracy expressions is modified to cover the case of correlated measurement noise and finally conclusions are drawn in Section 7.

\section{Parametric Identification}

In this section, three different approaches to identify a parametric model of the complex modulus are described. One approach is based on the nonparametric estimate described in for example $[8,10]$, while the other two fit the parametric model directly from the measured data. In all three cases, a least squares type loss function $J(\boldsymbol{\theta})$ is used, and an estimate of the parameter vector $\boldsymbol{\theta}$ is found through

$$
\hat{\boldsymbol{\theta}}=\arg \min _{\boldsymbol{\theta}} J(\boldsymbol{\theta}) .
$$

The theory does not depend on the model chosen for the complex modulus, and no model is therefore specified at this stage.

The theory in this report applies to longitudinal wave propagation in a slender bar, but can easily be modified to cover other types of experimental setups. In a longitudinal wave propagation, a slender bar of length $L$ is axially excited at one end as is seen in Fig. 1. The excitation gives rise to longitudinal strain waves traveling back and forth in the bar. This wave propagation can in the frequency domain be described by the so called frequency domain wave equation

$$
\frac{\partial^{2} \varepsilon(x, \omega)}{\partial x^{2}}-\gamma^{2}(\omega) \varepsilon(x, \omega)=0,
$$

with general solution

$$
\varepsilon(x, \omega)=P(\omega) \mathrm{e}^{-\gamma(\omega) x}+N(\omega) \mathrm{e}^{\gamma(\omega) x} .
$$

In (4), the frequency dependent functions $P(\omega)$ and $N(\omega)$ are the amplitudes of waves traveling in positive and negative $x$ direction, respectively. The function $\gamma(\omega)$ in $(3)$ and (4) is called the wave propagation function and is related to the complex modulus $E(\omega)$ through

$$
\gamma^{2}(\omega)=-\frac{\rho \omega^{2}}{E(\omega)}
$$


The strains caused by the excitation is measured at $n$ different points $\left\{x_{i}\right\}_{i=1}^{n}$ along the bar at $N$ discrete time instances and then transformed into frequency domain using the discrete Fourier transform (DFT). Using the strains from all $n$ sensors, the following system of equations can be formed

$$
\left[\begin{array}{c}
\varepsilon\left(\omega, x_{1}\right) \\
\vdots \\
\varepsilon\left(\omega, x_{n}\right)
\end{array}\right]=\left[\begin{array}{cc}
\mathrm{e}^{-\gamma(\omega) x_{1}} & \mathrm{e}^{\gamma(\omega) x_{n}} \\
\vdots & \vdots \\
\mathrm{e}^{-\gamma(\omega) x_{n}} & \mathrm{e}^{\gamma(\omega) x_{n}}
\end{array}\right]\left[\begin{array}{c}
P(\omega) \\
N(\omega)
\end{array}\right]=\mathbf{A}(\omega) \mathbf{c}(\omega),
$$

and used to identify the complex modulus. In the following sections it is shown how the frequency domain data described above can be used to fit a parametric model of the complex modulus.

\subsection{Parametric Identification Using the Nonparametric Estimate}

In [11], an approach to fit a parametric model based on the nonparametric estimate in $[8,10]$ is described. The loss function is in this case given by

$$
J(\boldsymbol{\theta})=\sum_{k=n_{\omega}}^{N_{\omega}} \frac{1}{C\left(\omega_{k}\right)}\left|\epsilon\left(\omega_{k}, \boldsymbol{\theta}\right)\right|^{2}
$$

where

$$
\epsilon(\omega, \boldsymbol{\theta})=\hat{E}(\omega)-E(\omega, \boldsymbol{\theta}) .
$$

In $(8), \hat{E}(\omega)$ is the nonparametric estimate in $[8,10]$ and $E(\omega, \boldsymbol{\theta})$ is the complex modulus model. Furthermore, the weighting $C\left(\omega_{k}\right)$ in $(7)$ is chosen as the variance of the nonparametric estimate, i.e.

$$
C\left(\omega_{k}\right)=\operatorname{var}\left\{\hat{E}\left(\omega_{k}\right)\right\}
$$

This is given by the covariance expression derived in [8]

$$
\operatorname{cov}\left\{\hat{E}\left(\omega_{k}\right), \hat{E}\left(\omega_{l}\right)\right\}=\frac{\lambda^{2}}{h\left(\omega_{k}\right)} \delta_{k, l}, \quad 0<k, l<\frac{N}{2},
$$

where $\lambda^{2}$ is the noise variance, $\delta_{k, l}$ is the Kronecker delta function, and $h(\omega)$ is given by

$$
h(\omega)=\mathbf{c}^{*}(\omega) \mathbf{A}_{\mathcal{R}}^{*}(\omega) \mathbf{P}(\omega) \mathbf{A}_{\mathcal{R}}(\omega) \mathbf{c}(\omega) .
$$

In (11), $\mathbf{A}_{\mathcal{R}}(\omega)$ is defined as the derivative of $\mathbf{A}(\omega)$ with respect to the real part of the complex modulus,

$$
\mathbf{P}(\omega)=\mathbf{I}_{n}-\mathbf{A}(\omega) \mathbf{A}^{\dagger}(\omega)
$$

is the orthogonal projection onto the null space of $\mathbf{A}^{*}(\omega)$, and $\mathbf{c}(\omega)$ and $\mathbf{A}(\omega)$ are defined through (6). Note that both $\mathbf{A}(\omega)$ and $\mathbf{P}(\omega)$ are implicit functions of the true complex modulus, $c f$. (6). The upper and lower limits, $n_{\omega}$ and $N_{\omega}$, of the sum in (7) are chosen so that $\left\{\omega_{k}\right\}_{k=n_{\omega}}^{N_{\omega}}$ coincide with the range of useful frequencies for the experiment. This range depends on the experimental setup, as is discussed in [10]. An estimate of the parameter vector, $\hat{\boldsymbol{\theta}}_{\mathrm{NP}}$, is obtained through (7) and (2). 


\subsection{Fitting a Parametric Model Directly from Data}

In Section 2.1, a parametric model was fitted based on a nonparametric estimate of the complex modulus. In [10], a parametric model is identified from the Fourier transformed data directly. The model is here fitted from $n-1$ of the $n$ strain measurements, while the $n$ :th strain measurement (together with a free end boundary condition at $x=0$ implying that the strain at this section is equal to zero) is used to identify the unknown amplitudes of the waves traveling back and forth in the bar. The loss function is hence given by

$$
J(\boldsymbol{\theta})=\sum_{k=n_{\omega}}^{N_{\omega}} \sum_{i=1}^{n-1}\left|\varepsilon_{\mathcal{M}}\left(\omega_{k}, x_{i}\right)-\varepsilon\left(\omega_{k}, x_{i}, \boldsymbol{\theta}\right)\right|^{2},
$$

where $n_{\omega}$ and $N_{\omega}$ are chosen as in Section 2.1, $\varepsilon_{\mathcal{M}}\left(\omega, x_{i}\right)$ is the measured strain at section $x_{i}$ and frequency $\omega$, and the data model $\varepsilon\left(\omega, x_{i}, \boldsymbol{\theta}\right)$ is given by

$$
\varepsilon\left(\omega, x_{i}, \boldsymbol{\theta}\right)=P(\omega) \mathrm{e}^{-\gamma(\omega, \boldsymbol{\theta}) x_{i}}+N(\omega) \mathrm{e}^{\gamma(\omega, \boldsymbol{\theta}) x_{i}},
$$

cf. (4). In (14), $P(\omega)$ and $N(\omega)$ are the unknown amplitudes of waves traveling in positive and negative $x$ direction, respectively. As was mentioned above, these are estimated from the free end boundary condition

$$
\varepsilon(\omega, 0, \boldsymbol{\theta})=0
$$

and an $n$ :th strain measurement not included in $J(\boldsymbol{\theta})$. Applying (15) to (14) gives $P(\omega)=-N(\omega)$ and hence

$$
\varepsilon\left(\omega, x_{i}, \boldsymbol{\theta}\right)=2 N(\omega) \sinh \left(\gamma(\omega, \boldsymbol{\theta}) x_{i}\right) .
$$

The amplitude $N(\omega)$ can now be estimated from (16) and the $n$ :th strain measurement according to

$$
\hat{N}(\omega, \boldsymbol{\theta})=\arg \min _{N(\omega)}\left|\varepsilon_{\mathcal{M}}\left(\omega, x_{n}\right)-\varepsilon\left(\omega, x_{n}, \boldsymbol{\theta}\right)\right|^{2}=\frac{\varepsilon_{\mathcal{M}}\left(\omega, x_{n}\right)}{2 \sinh \left(\gamma(\omega, \boldsymbol{\theta}) x_{n}\right)}
$$

Note that this estimate depends on the model parameters $\boldsymbol{\theta}$. The model output $\varepsilon\left(\omega, x_{i}, \boldsymbol{\theta}\right)$ in $(13)$ can now be replaced by

$$
\hat{\varepsilon}\left(\omega, x_{i}, \boldsymbol{\theta}\right)=2 \hat{N}(\omega, \boldsymbol{\theta}) \sinh \left(\gamma(\omega, \boldsymbol{\theta}) x_{i}\right)=\frac{\sinh \left(\gamma(\omega, \boldsymbol{\theta}) x_{i}\right)}{\sinh \left(\gamma(\omega, \boldsymbol{\theta}) x_{n}\right)} \varepsilon_{\mathcal{M}}\left(\omega, x_{n}\right),
$$

and the new loss function written as

$$
J(\boldsymbol{\theta})=\sum_{k=n_{\omega}}^{N_{\omega}} \sum_{i=1}^{n-1}\left|\varepsilon_{\mathcal{M}}\left(\omega_{k}, x_{i}\right)-\hat{\varepsilon}\left(\omega_{k}, x_{i}, \boldsymbol{\theta}\right)\right|^{2}=\sum_{k=n_{\omega}}^{N_{\omega}} \underline{\boldsymbol{\epsilon}}^{*}\left(\omega_{k}, \boldsymbol{\theta}\right) \underline{\boldsymbol{\epsilon}}\left(\omega_{k}, \boldsymbol{\theta}\right),
$$

where

$$
\underline{\boldsymbol{\epsilon}}(\omega, \boldsymbol{\theta})=\underline{\varepsilon}_{\mathcal{M}}(\omega)-S(\omega, \boldsymbol{\theta}) \varepsilon_{\mathcal{M}}\left(\omega, x_{n}\right) .
$$


In $(20), \underline{\varepsilon}_{\mathcal{M}}(\omega)$ and $S(\omega, \boldsymbol{\theta})$ are given by

$$
\begin{aligned}
\underline{\varepsilon}_{\mathcal{M}}(\omega) & =\left[\begin{array}{lll}
\varepsilon_{\mathcal{M}}\left(\omega, x_{1}\right) & \cdots & \varepsilon_{\mathcal{M}}\left(\omega, x_{n-1}\right)
\end{array}\right]^{T} \\
S(\omega, \boldsymbol{\theta}) & =\frac{1}{\sinh \left(\gamma(\omega, \boldsymbol{\theta}) x_{n}\right)}\left[\begin{array}{lll}
\sinh \left(\gamma(\omega, \boldsymbol{\theta}) x_{1}\right) & \cdots & \sinh \left(\gamma(\omega, \boldsymbol{\theta}) x_{n-1}\right)
\end{array}\right]^{T} .
\end{aligned}
$$

An estimate of the parameter vector, $\hat{\boldsymbol{\theta}}_{\mathrm{D}}$, is in this case obtained through (19) and (2).

\subsection{Fitting a Parametric Model Directly from Data - A Modified Approach}

In the previous section, an approach to finding a parametric estimate of the complex modulus straight from the Fourier transformed data was described. In this approach, the unknown amplitudes $P(\omega)$ and $N(\omega)$ were identified from a free end boundary condition and one strain measurement. All the knowledge of the material response is hence not included when estimating these amplitudes. In this section, a modified approach is proposed where all knowledge of the material response is included both in estimating the unknown amplitudes and in fitting the model parameters. The loss function is in this case given by

$$
J(\boldsymbol{\theta})=\sum_{k=n_{\omega}}^{N_{\omega}} \sum_{i=1}^{n}\left|\varepsilon_{\mathcal{M}}\left(\omega_{k}, x_{i}\right)-\varepsilon\left(\omega_{k}, x_{i}, \boldsymbol{\theta}\right)\right|^{2}=\sum_{k=n_{\omega}}^{N_{\omega}}\left\|\varepsilon_{\mathcal{M}}\left(\omega_{k}\right)-\varepsilon\left(\omega_{k}, \boldsymbol{\theta}\right)\right\|^{2},
$$

where $n_{\omega}$ and $N_{\omega}$ are chosen as in Section 2.1, and

$$
\begin{aligned}
\varepsilon_{\mathcal{M}}\left(\omega_{k}\right) & =\left[\begin{array}{lll}
\varepsilon_{\mathcal{M}}\left(\omega_{k}, x_{1}\right) & \cdots & \varepsilon_{\mathcal{M}}\left(\omega_{k}, x_{n}\right)
\end{array}\right]^{T} \\
\varepsilon(\omega, \boldsymbol{\theta}) & =\left[\begin{array}{lll}
\varepsilon\left(\omega, x_{1}, \boldsymbol{\theta}\right) & \cdots & \varepsilon\left(\omega, x_{n}, \boldsymbol{\theta}\right)
\end{array}\right]^{T} .
\end{aligned}
$$

The data model vector $\boldsymbol{\varepsilon}(\omega, \boldsymbol{\theta})$ can, by using (14), be written as

$$
\begin{aligned}
\varepsilon(\omega, \boldsymbol{\theta}) & =\left[\begin{array}{cc}
\mathrm{e}^{-\gamma(\omega, \boldsymbol{\theta}) x_{1}} & \mathrm{e}^{\gamma(\omega, \boldsymbol{\theta}) x_{n}} \\
\vdots & \vdots \\
\mathrm{e}^{-\gamma(\omega, \boldsymbol{\theta}) x_{n}} & \mathrm{e}^{\gamma(\omega, \boldsymbol{\theta}) x_{n}}
\end{array}\right]\left[\begin{array}{c}
P(\omega) \\
N(\omega)
\end{array}\right] \\
& \triangleq \mathbf{A}(\omega, \boldsymbol{\theta}) \mathbf{c}(\omega),
\end{aligned}
$$

cf. (6). The amplitudes $P(\omega)$ and $N(\omega)$ can now be estimated as

$$
\begin{aligned}
\hat{\mathbf{c}}(\omega, \boldsymbol{\theta}) & =\arg \min _{\mathbf{c}(\omega)}\left\|\varepsilon_{\mathcal{M}}(\omega)-\varepsilon(\omega, \boldsymbol{\theta})\right\|^{2} \\
& =\mathbf{A}^{\dagger}(\omega, \boldsymbol{\theta}) \varepsilon_{\mathcal{M}}(\omega),
\end{aligned}
$$

where $\mathbf{A}^{\dagger}(\omega, \boldsymbol{\theta})$ is the pseudo-inverse of $\mathbf{A}(\omega, \boldsymbol{\theta})$. Replacing the data model vector $\boldsymbol{\varepsilon}(\omega, \boldsymbol{\theta})$ in (23) by the corresponding vector

$$
\hat{\varepsilon}(\omega, \boldsymbol{\theta})=\mathbf{A}(\omega, \boldsymbol{\theta}) \hat{\mathbf{c}}(\omega, \boldsymbol{\theta})=\mathbf{A}(\omega, \boldsymbol{\theta}) \mathbf{A}^{\dagger}(\omega, \boldsymbol{\theta}) \varepsilon_{\mathcal{M}}(\omega),
$$


the loss function can be rewritten as

$$
\begin{aligned}
J(\boldsymbol{\theta}) & =\sum_{k=n_{\omega}}^{N_{\omega}}\left\|\varepsilon_{\mathcal{M}}\left(\omega_{k}\right)-\mathbf{A}\left(\omega_{k}, \boldsymbol{\theta}\right) \mathbf{A}^{\dagger}\left(\omega_{k}, \boldsymbol{\theta}\right) \varepsilon_{\mathcal{M}}\left(\omega_{k}\right)\right\|^{2} \\
& =\sum_{k=n_{\omega}}^{N_{\omega}} \varepsilon_{\mathcal{M}}^{*}\left(\omega_{k}\right) \mathbf{P}\left(\omega_{k}, \boldsymbol{\theta}\right) \varepsilon_{\mathcal{M}}\left(\omega_{k}\right)
\end{aligned}
$$

where $\mathbf{P}(\omega, \boldsymbol{\theta})$ is the orthogonal projection operator onto the null space of $\mathbf{A}^{*}(\omega, \boldsymbol{\theta}), c f . \quad(12)$. An estimate of the parameter vector, $\hat{\boldsymbol{\theta}}_{\mathrm{DM}}$, can now be obtained through (29) and (2).

\section{Accuracy Analysis}

In this section, asymptotic expressions for the accuracy of the parametric estimates described in Section 2 are derived. These are valid when the signalto-noise ratio (SNR) is large, which is typically the case in the application considered here. An expression for the accuracy of the nonparametric based estimate is outlined in [11], but will here be presented in more detail.

First, a general expression for the correlation between the estimation errors of the parameter vector is derived. Recall that in all three cases, $\hat{\boldsymbol{\theta}}$ is a minimum of $J(\boldsymbol{\theta})$ and hence

$$
J_{\boldsymbol{\theta}}^{\prime}(\hat{\boldsymbol{\theta}})=\mathbf{0} .
$$

Approximating $J_{\boldsymbol{\theta}}^{\prime}(\boldsymbol{\theta})$ by the truncated Taylor series expansion around the true parameter vector $\boldsymbol{\theta}_{0}$, and evaluating for $\boldsymbol{\theta}=\hat{\boldsymbol{\theta}}$, gives

$$
\mathbf{0}=J_{\boldsymbol{\theta}}^{\prime}(\hat{\boldsymbol{\theta}}) \approx J_{\boldsymbol{\theta}}^{\prime}\left(\boldsymbol{\theta}_{0}\right)+\left(\hat{\boldsymbol{\theta}}-\boldsymbol{\theta}_{0}\right)^{T} J_{\boldsymbol{\theta}}^{\prime \prime}\left(\boldsymbol{\theta}_{0}\right)
$$

The estimation error $\tilde{\boldsymbol{\theta}}$ is hence given by

$$
\tilde{\boldsymbol{\theta}}^{T}=\left(\hat{\boldsymbol{\theta}}-\boldsymbol{\theta}_{0}\right)^{T} \approx-J_{\boldsymbol{\theta}}^{\prime}\left(\boldsymbol{\theta}_{0}\right)\left[J_{\boldsymbol{\theta}}^{\prime \prime}\left(\boldsymbol{\theta}_{0}\right)\right]^{-1}
$$

Using (32), the accuracy of the estimated parameter vector can, for large SNR, be approximated by

$$
\mathbb{E}\left\{\tilde{\boldsymbol{\theta}} \tilde{\boldsymbol{\theta}}^{T}\right\} \approx \mathcal{H}^{-1} \mathcal{G} \mathcal{H}^{-1}
$$

where

$$
\begin{aligned}
\mathcal{G} & =\mathbb{E}\left\{\left[J_{\boldsymbol{\theta}}^{\prime}\left(\boldsymbol{\theta}_{0}\right)\right]^{T} J_{\boldsymbol{\theta}}^{\prime}\left(\boldsymbol{\theta}_{0}\right)\right\} \\
\mathcal{H} & =\lim _{\mathrm{SNR} \rightarrow \infty} J_{\boldsymbol{\theta}}^{\prime \prime}\left(\boldsymbol{\theta}_{0}\right) .
\end{aligned}
$$

The accuracy of the corresponding complex modulus can also be obtained from the expression in (33). Let

$$
\mathbf{e}(\omega, \boldsymbol{\theta})=\left[\begin{array}{ll}
E_{\mathcal{R}}(\omega, \boldsymbol{\theta}) & E_{\mathcal{I}}(\omega, \boldsymbol{\theta})
\end{array}\right]^{T},
$$


where

$$
E(\omega, \boldsymbol{\theta})=E_{\mathcal{R}}(\omega, \boldsymbol{\theta})+\mathrm{i} E_{\mathcal{I}}(\omega, \boldsymbol{\theta}) .
$$

Linearization of $\mathbf{e}(\omega, \boldsymbol{\theta})$ around $\boldsymbol{\theta}_{0}$, and evaluating at $\boldsymbol{\theta}=\hat{\boldsymbol{\theta}}$, gives

$$
\mathbf{e}(\omega, \hat{\boldsymbol{\theta}}) \approx \mathbf{e}\left(\omega, \boldsymbol{\theta}_{0}\right)+\frac{\mathrm{d}}{\mathrm{d} \boldsymbol{\theta}} \mathbf{e}\left(\omega, \boldsymbol{\theta}_{0}\right)\left(\hat{\boldsymbol{\theta}}-\boldsymbol{\theta}_{0}\right)
$$

and hence

$$
\begin{aligned}
\mathbb{E}\left\{\tilde{\mathbf{e}}\left(\omega_{k}, \hat{\boldsymbol{\theta}}\right) \tilde{\mathbf{e}}^{T}\left(\omega_{l}, \hat{\boldsymbol{\theta}}\right)\right\} \\
\quad=\mathbb{E}\left\{\left(\mathbf{e}\left(\omega_{k}, \hat{\boldsymbol{\theta}}\right)-\mathbf{e}\left(\omega_{k}, \boldsymbol{\theta}_{0}\right)\right)\left(\mathbf{e}\left(\omega_{l}, \hat{\boldsymbol{\theta}}\right)-\mathbf{e}\left(\omega_{l}, \boldsymbol{\theta}_{0}\right)\right)^{T}\right\} \\
\quad \approx\left[\frac{\mathrm{d}}{\mathrm{d} \boldsymbol{\theta}} \mathbf{e}\left(\omega_{k}, \boldsymbol{\theta}_{0}\right)\right] \mathbb{E}\left\{\tilde{\boldsymbol{\theta}} \tilde{\boldsymbol{\theta}}^{T}\right\}\left[\frac{\mathrm{d}}{\mathrm{d} \boldsymbol{\theta}} \mathbf{e}\left(\omega_{l}, \boldsymbol{\theta}_{0}\right)\right]^{T}
\end{aligned}
$$

In the following, explicit expressions for $\mathcal{G}$ and $\mathcal{H}$ are derived for the three different cases described in the previous section.

\subsection{Accuracy Analysis of the Nonparametric Based Esti- mate}

In this analysis, it is assumed that the material can be described by the chosen model, so that the nonparametric estimate is given by

$$
\hat{E}(\omega)=E\left(\omega, \boldsymbol{\theta}_{0}\right)+\tilde{E}(\omega),
$$

and that

$$
\lim _{\mathrm{SNR} \rightarrow \infty} \hat{E}(\omega)=E\left(\omega, \boldsymbol{\theta}_{0}\right)
$$

In $(40), \boldsymbol{\theta}_{0}$ is the true parameter vector and $\tilde{E}(\omega)$ is a stochastic disturbance. The assumption in (41) asserts that this disturbance goes to zero when the SNR tends to infinity, which corresponds to noise-free measurements of the strains. Furthermore, based on (10), we have that

$$
\begin{aligned}
& \mathbb{E}\left\{\tilde{E}\left(\omega_{k}\right) \tilde{E}^{*}\left(\omega_{l}\right)\right\}= \begin{cases}\frac{\lambda^{2}}{h\left(\omega_{k}\right)}=C\left(\omega_{k}\right) & k=l \\
0 & k \neq l\end{cases} \\
& \mathbb{E}\left\{\tilde{E}\left(\omega_{k}\right) \tilde{E}\left(\omega_{l}\right)\right\}=0 \forall k, l .
\end{aligned}
$$

Differentiating (7) with respect to $\boldsymbol{\theta}$, and evaluating for $\boldsymbol{\theta}=\boldsymbol{\theta}_{0}$, gives

$$
\begin{aligned}
J_{\boldsymbol{\theta}}^{\prime}\left(\boldsymbol{\theta}_{0}\right) & =\sum_{k=n_{\omega}}^{N_{\omega}} \frac{1}{C\left(\omega_{k}\right)}\left[\epsilon\left(\omega_{k}, \boldsymbol{\theta}_{0}\right) \Psi^{*}\left(\omega_{k}, \boldsymbol{\theta}_{0}\right)+\epsilon^{*}\left(\omega_{k}, \boldsymbol{\theta}_{0}\right) \Psi^{T}\left(\omega_{k}, \boldsymbol{\theta}_{0}\right)\right] \\
& =\sum_{k=n_{\omega}}^{N_{\omega}} \frac{1}{C\left(\omega_{k}\right)}\left[\tilde{E}\left(\omega_{k}\right) \Psi^{*}\left(\omega_{k}, \boldsymbol{\theta}_{0}\right)+\tilde{E}^{*}\left(\omega_{k}\right) \Psi^{T}\left(\omega_{k}, \boldsymbol{\theta}_{0}\right)\right]
\end{aligned}
$$


where

$$
\Psi(\omega, \boldsymbol{\theta})=\left[\frac{\mathrm{d}}{\mathrm{d} \boldsymbol{\theta}} \epsilon(\omega, \boldsymbol{\theta})\right]^{T}=-\left[\frac{\mathrm{d}}{\mathrm{d} \boldsymbol{\theta}} E(\omega, \boldsymbol{\theta})\right]^{T}
$$

is the derivative of $\epsilon(\omega, \boldsymbol{\theta})$ with respect to $\boldsymbol{\theta}=\left[\begin{array}{lll}\theta_{1} & \cdots & \theta_{m}\end{array}\right]^{T}$. In the second equality of (43), we have used that

$$
\epsilon\left(\omega, \boldsymbol{\theta}_{0}\right)=\hat{E}(\omega)-E\left(\omega, \boldsymbol{\theta}_{0}\right)=\tilde{E}(\omega)
$$

which follows from $(40)$ and (8). The matrix $\mathcal{G}_{\mathrm{NP}}$ can now be evaluated as

$$
\begin{aligned}
& \mathcal{G}_{\mathrm{NP}}=\mathbb{E}\left\{\left[J_{\boldsymbol{\theta}}^{\prime}\left(\boldsymbol{\theta}_{0}\right)\right]^{T} J_{\boldsymbol{\theta}}^{\prime}\left(\boldsymbol{\theta}_{0}\right)\right\} \\
&=\sum_{k=n_{\omega}}^{N_{\omega}} \frac{1}{C^{2}\left(\omega_{k}\right)}\left[C\left(\omega_{k}\right) \Psi\left(\omega_{k}, \boldsymbol{\theta}_{0}\right) \Psi^{*}\left(\omega_{k}, \boldsymbol{\theta}_{0}\right)\right. \\
&\left.\quad+C\left(\omega_{k}\right)\left[\Psi\left(\omega_{k}, \boldsymbol{\theta}_{0}\right) \Psi^{*}\left(\omega_{k}, \boldsymbol{\theta}_{0}\right)\right]^{T}\right] \\
&=\sum_{k=n_{\omega}}^{N_{\omega}} \frac{1}{C\left(\omega_{k}\right)}\left[\Psi\left(\omega_{k}, \boldsymbol{\theta}_{0}\right) \Psi^{*}\left(\omega_{k}, \boldsymbol{\theta}_{0}\right)+\left[\Psi\left(\omega_{k}, \boldsymbol{\theta}_{0}\right) \Psi^{*}\left(\omega_{k}, \boldsymbol{\theta}_{0}\right)\right]^{T}\right]
\end{aligned}
$$

The second derivative can be evaluated in a similar fashion, giving

$$
\begin{aligned}
J_{\boldsymbol{\theta}}^{\prime \prime}\left(\boldsymbol{\theta}_{0}\right)= & \sum_{k=n_{\omega}}^{N_{\omega}} \frac{1}{C\left(\omega_{k}\right)}\left[\Psi\left(\omega_{k}, \boldsymbol{\theta}_{0}\right) \Psi^{*}\left(\omega_{k}, \boldsymbol{\theta}_{0}\right)\right. \\
& +\left[\Psi\left(\omega_{k}, \boldsymbol{\theta}_{0}\right) \Psi^{*}\left(\omega_{k}, \boldsymbol{\theta}_{0}\right)\right]^{T}+\tilde{E}\left(\omega_{k}\right)\left[\frac{\mathrm{d}^{2}}{\mathrm{~d} \boldsymbol{\theta}^{2}} \epsilon^{*}\left(\omega_{k}, \boldsymbol{\theta}_{0}\right)\right] \\
& \left.+\tilde{E}^{*}\left(\omega_{k}\right)\left[\frac{\mathrm{d}^{2}}{\mathrm{~d} \boldsymbol{\theta}^{2}} \epsilon\left(\omega_{k}, \boldsymbol{\theta}_{0}\right)\right]\right]
\end{aligned}
$$

and

$$
\begin{aligned}
\mathcal{H}_{\mathrm{NP}} & =\lim _{\mathrm{SNR} \rightarrow \infty} J_{\boldsymbol{\theta}}^{\prime \prime}\left(\boldsymbol{\theta}_{0}\right) \\
& =\sum_{k=n_{\omega}}^{N_{\omega}} \frac{1}{C\left(\omega_{k}\right)}\left[\Psi\left(\omega_{k}, \boldsymbol{\theta}_{0}\right) \Psi^{*}\left(\omega_{k}, \boldsymbol{\theta}_{0}\right)+\left[\Psi\left(\omega_{k}, \boldsymbol{\theta}_{0}\right) \Psi^{*}\left(\omega_{k}, \boldsymbol{\theta}_{0}\right)\right]^{T}\right]=\mathcal{G}_{\mathrm{NP}}
\end{aligned}
$$

\subsection{Accuracy Analysis of the Parametric Estimate Iden- tified Directly from Data}

In this analysis, it is assumed that the data can be perfectly modeled by the wave propagation model in (14). As a consequence

$$
\varepsilon_{\mathcal{M}}\left(\omega, x_{i}\right)=\varepsilon\left(\omega, x_{i}, \boldsymbol{\theta}_{0}\right)+v\left(\omega, x_{i}\right)
$$

where $v(\omega)$ denotes the frequency domain measurement noise. The measurement noise is in the frequency domain assumed to be a circular ${ }^{1}$ white $\mathcal{C N}\left(0, \lambda^{2}\right)$

\footnotetext{
${ }^{1} \mathrm{~A}$ complex valued sequence $\{v(a)\}$ is called circular if its real and imaginary parts are mutually independent and identically distributed, i.e. $\mathbb{E}\{v(a) v(b)\}=0 \quad \forall a, b$.
} 
sequence, which is uncorrelated between both frequencies and between sensors. This assumption is exactly fulfilled if the time domain noise is a temporally and spatially white Gaussian noise process, and approximately fulfilled for any white noise process with identically distributed parameters and existing moments of any order, see [13]. It is also assumed that

$$
\lim _{\mathrm{SNR} \rightarrow \infty} \hat{N}\left(\omega, \boldsymbol{\theta}_{0}\right)=N(\omega)=\frac{\varepsilon\left(\omega, x_{n}, \boldsymbol{\theta}_{0}\right)}{2 \sinh \left(\gamma\left(\omega, \boldsymbol{\theta}_{0}\right) x_{n}\right)} .
$$

Before continuing the analysis, the following property and notation are introduced.

Property 1: The model error $\underline{\boldsymbol{\epsilon}}(\omega, \boldsymbol{\theta})$ in $(20)$ evaluated at $\boldsymbol{\theta}=\boldsymbol{\theta}_{0}$ is given by

$$
\begin{aligned}
\underline{\boldsymbol{\epsilon}}\left(\omega, \boldsymbol{\theta}_{0}\right) & =\underline{\boldsymbol{\varepsilon}}_{\mathcal{M}}(\omega)-S\left(\omega, \boldsymbol{\theta}_{0}\right) \varepsilon_{\mathcal{M}}\left(\omega, x_{n}\right) \\
& =\underline{\boldsymbol{\varepsilon}}\left(\omega, \boldsymbol{\theta}_{0}\right)+\underline{\mathbf{v}}(\omega)-S\left(\omega, \boldsymbol{\theta}_{0}\right)\left[\varepsilon\left(\omega, x_{n}, \boldsymbol{\theta}_{0}\right)+v\left(\omega, x_{n}\right)\right] \\
& =\underline{\mathbf{v}}(\omega)-S\left(\omega, \boldsymbol{\theta}_{0}\right) v\left(\omega, x_{n}\right)
\end{aligned}
$$

where

$$
\begin{aligned}
\underline{\varepsilon}\left(\omega, \boldsymbol{\theta}_{0}\right) & =\left[\begin{array}{lll}
\varepsilon\left(\omega, x_{1}, \boldsymbol{\theta}_{0}\right) & \cdots & \varepsilon\left(\omega, x_{n-1}, \boldsymbol{\theta}_{0}\right)
\end{array}\right]^{T} \\
\underline{\mathbf{v}}(\omega) & =\left[\begin{array}{lll}
v\left(\omega, x_{1}\right) & \cdots & v\left(\omega, x_{n-1}\right)
\end{array}\right]^{T} .
\end{aligned}
$$

In the third equality of (51), the property

$$
S\left(\omega, \boldsymbol{\theta}_{0}\right) \varepsilon\left(\omega, x_{n}, \boldsymbol{\theta}_{0}\right)=\underline{\varepsilon}\left(\omega, \boldsymbol{\theta}_{0}\right)
$$

has been used, which follows from (16), (22) and (50).

Notation 1: Let the derivatives of $S(\omega, \boldsymbol{\theta})$ and $\underline{\boldsymbol{\epsilon}}(\omega, \boldsymbol{\theta})$ with respect to $\boldsymbol{\theta}=$ $\left[\begin{array}{lll}\theta_{1} & \cdots & \theta_{m}\end{array}\right]^{T}$ be given by

$$
\Pi_{S}(\omega, \boldsymbol{\theta})=-\left[\frac{\mathrm{d}}{\mathrm{d} \boldsymbol{\theta}} S(\omega, \boldsymbol{\theta})\right]^{T}
$$

and

$$
\begin{aligned}
\Pi(\omega, \boldsymbol{\theta}) & =\left[\frac{\mathrm{d}}{\mathrm{d} \boldsymbol{\theta}} \underline{\boldsymbol{\epsilon}}(\omega, \boldsymbol{\theta})\right]^{T}=-\Pi_{S}(\omega, \boldsymbol{\theta}) \varepsilon_{\mathcal{M}}\left(\omega, x_{n}\right) \\
& =-\Pi_{S}(\omega, \boldsymbol{\theta})\left[\varepsilon\left(\omega, x_{n}, \boldsymbol{\theta}_{0}\right)+v\left(\omega, x_{n}\right)\right] .
\end{aligned}
$$

Both $\Pi_{S}(\cdot)$ and $\Pi(\cdot)$ are here $m \times(n-1)$ matrices.

The derivative of the loss function, evaluated at $\boldsymbol{\theta}=\boldsymbol{\theta}_{0}$, can now be written as

$$
J_{\boldsymbol{\theta}}^{\prime}\left(\boldsymbol{\theta}_{0}\right)=\sum_{k=n_{\omega}}^{N_{\omega}}\left[\underline{\boldsymbol{\epsilon}}^{*}\left(\omega_{k}, \boldsymbol{\theta}_{0}\right) \Pi^{T}\left(\omega_{k}, \boldsymbol{\theta}_{0}\right)+\underline{\boldsymbol{\epsilon}}^{T}\left(\omega_{k}, \boldsymbol{\theta}_{0}\right) \Pi^{*}\left(\omega_{k}, \boldsymbol{\theta}_{0}\right)\right]
$$


Through $(57), \mathcal{G}_{\mathrm{D}}$ can then be evaluated as

$$
\begin{aligned}
\mathcal{G}_{\mathrm{D}} & =\mathbb{E}\left\{\left[J_{\boldsymbol{\theta}}^{\prime}\left(\boldsymbol{\theta}_{0}\right)\right]^{T} J_{\boldsymbol{\theta}}^{\prime}\left(\boldsymbol{\theta}_{0}\right)\right\} \\
= & \sum_{k=n_{\omega}}^{N_{\omega}} \sum_{l=n_{\omega}}^{N_{\omega}} \mathbb{E}\left\{\left[\underline{\epsilon}^{*}\left(\omega_{k}, \boldsymbol{\theta}_{0}\right) \Pi^{T}\left(\omega_{k}, \boldsymbol{\theta}_{0}\right)+\underline{\boldsymbol{\epsilon}}^{T}\left(\omega_{k}, \boldsymbol{\theta}_{0}\right) \Pi^{*}\left(\omega_{k}, \boldsymbol{\theta}_{0}\right)\right]^{T}\right. \\
& \times\left[\underline{\boldsymbol{\epsilon}}^{*}\left(\omega_{l}, \boldsymbol{\theta}_{0}\right) \Pi^{T}\left(\omega_{l}, \boldsymbol{\theta}_{0}\right)+\underline{\boldsymbol{\epsilon}}^{T}\left(\omega_{l}, \boldsymbol{\theta}_{0}\right) \Pi^{*}\left(\omega_{l}, \boldsymbol{\theta}_{0}\right)\right] \\
= & \lambda^{2} \sum_{k=n_{\omega}}^{N_{\omega}}\left|\varepsilon\left(\omega_{k}, x_{n}, \boldsymbol{\theta}_{0}\right)\right|^{2}\left[\Pi_{S}\left(\omega_{k}, \boldsymbol{\theta}_{0}\right) \Pi_{S}^{*}\left(\omega_{k}, \boldsymbol{\theta}_{0}\right)\right. \\
& +\left[\Pi_{S}\left(\omega_{k}, \boldsymbol{\theta}_{0}\right) \Pi_{S}^{*}\left(\omega_{k}, \boldsymbol{\theta}_{0}\right)\right]^{T} \\
& +\Pi_{S}\left(\omega_{k}, \boldsymbol{\theta}_{0}\right)\left[S\left(\omega_{k}, \boldsymbol{\theta}_{0}\right) S^{*}\left(\omega_{k}, \boldsymbol{\theta}_{0}\right)\right]^{T} \Pi_{S}^{*}\left(\omega_{k}, \boldsymbol{\theta}_{0}\right) \\
& \left.+\left[\Pi_{S}\left(\omega_{k}, \boldsymbol{\theta}_{0}\right)\left[S\left(\omega_{k}, \boldsymbol{\theta}_{0}\right) S^{*}\left(\omega_{k}, \boldsymbol{\theta}_{0}\right)\right]^{T} \Pi_{S}^{*}\left(\omega_{k}, \boldsymbol{\theta}_{0}\right)\right]^{T}\right] .
\end{aligned}
$$

In the third equality of (58), the forth order moment of the noise has been neglected. This step also involves straightforward but tedious calculations, which will not be presented here. The derivation include replacing $\underline{\boldsymbol{\epsilon}}\left(\omega, \boldsymbol{\theta}_{0}\right)$ and $\Pi\left(\omega, \boldsymbol{\theta}_{0}\right)$ by (51) and (56), respectively, and using the noise assumptions giving

$$
\begin{aligned}
& \mathbb{E}\left\{\underline{\mathbf{v}}\left(\omega_{k}\right) \underline{\mathbf{y}}^{*}\left(\omega_{l}\right)\right\}=\lambda^{2} \mathbf{I}_{n-1} \delta_{k, l}, \quad 0<k, l<\frac{N}{2} \\
& \mathbb{E}\left\{\underline{\mathbf{v}}\left(\omega_{k}\right) \underline{\mathbf{v}}^{T}\left(\omega_{l}\right)\right\}=\mathbf{0} \quad \forall k, l
\end{aligned}
$$

and

$$
\begin{aligned}
\mathbb{E}\left\{v\left(\omega_{k}, x_{n}\right) \underline{\mathbf{v}}^{*}\left(\omega_{l}\right)\right\}=\mathbf{0}, & \forall k, l \\
\mathbb{E}\left\{v\left(\omega_{k}, x_{n}\right) \underline{\mathbf{v}}^{T}\left(\omega_{l}\right)\right\}=\mathbf{0}, & \forall k, l .
\end{aligned}
$$

Evaluating the second derivative in a similar fashion as (57) gives

$$
\begin{aligned}
J_{\boldsymbol{\theta}}^{\prime \prime}\left(\boldsymbol{\theta}_{0}\right)= & \sum_{k=n_{\omega}}^{N_{\omega}}\left[\Pi\left(\omega_{k}, \boldsymbol{\theta}_{0}\right) \Pi^{*}\left(\omega_{k}, \boldsymbol{\theta}_{0}\right)\right. \\
& +\left[\Pi\left(\omega_{k}, \boldsymbol{\theta}_{0}\right) \Pi^{*}\left(\omega_{k}, \boldsymbol{\theta}_{0}\right)\right]^{T}+\underline{\boldsymbol{\epsilon}}^{*}\left(\omega_{k}, \boldsymbol{\theta}_{0}\right) \frac{d}{d \boldsymbol{\theta}} \Pi^{T}\left(\omega_{k}, \boldsymbol{\theta}_{0}\right) \\
& \left.+\underline{\boldsymbol{\epsilon}}^{T}\left(\omega_{k}, \boldsymbol{\theta}_{0}\right) \frac{d}{d \boldsymbol{\theta}} \Pi^{*}\left(\omega_{k}, \boldsymbol{\theta}_{0}\right)\right] .
\end{aligned}
$$

When the SNR tends to infinity, corresponding to noise-free measurements of the strains, we have that

$$
\begin{aligned}
\lim _{\mathrm{SNR} \rightarrow \infty} \underline{\boldsymbol{\epsilon}}\left(\omega, \boldsymbol{\theta}_{0}\right) & =\mathbf{0} \\
\lim _{\mathrm{SNR} \rightarrow \infty} \Pi\left(\omega, \boldsymbol{\theta}_{0}\right) & =-\Pi_{S}\left(\omega, \boldsymbol{\theta}_{0}\right) \varepsilon\left(\omega, x_{n}, \boldsymbol{\theta}_{0}\right),
\end{aligned}
$$


which follows from (51) and (56), respectively. Using (61) and (62), $\mathcal{H}_{\mathrm{D}}$ can now be evaluated as

$$
\begin{aligned}
\mathcal{H}_{\mathrm{D}} & =\lim _{\mathrm{SNR} \rightarrow \infty} J_{\boldsymbol{\theta}}^{\prime \prime}\left(\boldsymbol{\theta}_{0}\right) \\
& =\sum_{k=n_{\omega}}^{N_{\omega}}\left|\varepsilon\left(\omega_{k}, x_{n}, \boldsymbol{\theta}_{0}\right)\right|^{2}\left[\Pi_{S}\left(\omega_{k}, \boldsymbol{\theta}_{0}\right) \Pi_{S}^{*}\left(\omega_{k}, \boldsymbol{\theta}_{0}\right)+\left[\Pi_{S}\left(\omega_{k}, \boldsymbol{\theta}_{0}\right) \Pi_{S}^{*}\left(\omega_{k}, \boldsymbol{\theta}_{0}\right)\right]^{T}\right] .
\end{aligned}
$$

\subsection{Accuracy Analysis of the Modified Data Approach}

In this analysis, the same assumptions as in Section 3.2 are made on the data. The measured data is hence assumed to be described by (49), and the frequency domain noise to be circular white $\mathcal{C N}\left(0, \lambda^{2}\right)$ sequence, uncorrelated between both frequencies and between sensors. Before evaluating $\mathcal{G}$ and $\mathcal{H}$, the following notations and properties are introduced.

Notation 2: Derivatives with respect to the elements in $\boldsymbol{\theta}=\left[\begin{array}{lll}\theta_{1} & \cdots & \theta_{m}\end{array}\right]^{T}$ are given by

$$
\begin{aligned}
\mathbf{A}_{i}(\omega, \boldsymbol{\theta}) & =\frac{\partial \mathbf{A}(\omega, \boldsymbol{\theta})}{\partial \theta_{i}} \\
\mathbf{A}_{i j}(\omega, \boldsymbol{\theta}) & =\frac{\partial^{2} \mathbf{A}(\omega, \boldsymbol{\theta})}{\partial \theta_{i} \partial \theta_{j}}
\end{aligned}
$$

Similar notation will be used to express the derivatives of any other function of $\boldsymbol{\theta}$.

Property 2: The following holds

$$
\begin{aligned}
& \varepsilon^{*}\left(\omega, \boldsymbol{\theta}_{0}\right) \mathbf{P}_{i}\left(\omega, \boldsymbol{\theta}_{0}\right) \mathbf{P}_{j}\left(\omega, \boldsymbol{\theta}_{0}\right) \boldsymbol{\varepsilon}\left(\omega, \boldsymbol{\theta}_{0}\right) \\
& =\mathbf{c}^{*}(\omega) \mathbf{A}_{i}^{*}\left(\omega, \boldsymbol{\theta}_{0}\right) \mathbf{P}\left(\omega, \boldsymbol{\theta}_{0}\right) \mathbf{A}_{j}\left(\omega, \boldsymbol{\theta}_{0}\right) \mathbf{c}\left(\omega, \boldsymbol{\theta}_{0}\right)
\end{aligned}
$$

and

$$
\begin{aligned}
\varepsilon^{*}\left(\omega, \boldsymbol{\theta}_{0}\right) \mathbf{P}_{i j}\left(\omega, \boldsymbol{\theta}_{0}\right) \boldsymbol{\varepsilon}\left(\omega, \boldsymbol{\theta}_{0}\right)= & \mathbf{c}^{*}(\omega) \mathbf{A}_{i}^{*}\left(\omega, \boldsymbol{\theta}_{0}\right) \mathbf{P}\left(\omega, \boldsymbol{\theta}_{0}\right) \mathbf{A}_{j}\left(\omega, \boldsymbol{\theta}_{0}\right) \mathbf{c}\left(\omega, \boldsymbol{\theta}_{0}\right) \\
& +\mathbf{c}^{*}(\omega) \mathbf{A}_{j}^{*}\left(\omega, \boldsymbol{\theta}_{0}\right) \mathbf{P}\left(\omega, \boldsymbol{\theta}_{0}\right) \mathbf{A}_{i}\left(\omega, \boldsymbol{\theta}_{0}\right) \mathbf{c}\left(\omega, \boldsymbol{\theta}_{0}\right),
\end{aligned}
$$

where $\mathbf{c}(\omega)$ is the vector of unknown amplitudes defined through (26).

Proof: See Appendix A.

Property 3: Neglecting the fourth order moment of the noise we have that

$$
\begin{aligned}
& \mathbb{E}\left\{\varepsilon_{\mathcal{M}}^{*}\left(\omega_{k}\right) \mathbf{P}_{i}\left(\omega_{k}, \boldsymbol{\theta}_{0}\right) \varepsilon_{\mathcal{M}}\left(\omega_{k}\right) \varepsilon_{\mathcal{M}}^{*}\left(\omega_{l}\right) \mathbf{P}_{j}\left(\omega_{l}, \boldsymbol{\theta}_{0}\right) \varepsilon_{\mathcal{M}}\left(\omega_{l}\right)\right\} \\
& \quad=\left\{\begin{array}{cl}
\lambda^{2} \varepsilon^{*}\left(\omega_{k}, \boldsymbol{\theta}_{0}\right)\left[\mathbf{P}_{i}\left(\omega_{k}, \boldsymbol{\theta}_{0}\right) \mathbf{P}_{j}\left(\omega_{k}, \boldsymbol{\theta}_{0}\right)\right. \\
\left.+\mathbf{P}_{j}\left(\omega_{k}, \boldsymbol{\theta}_{0}\right) \mathbf{P}_{i}\left(\omega_{k}, \boldsymbol{\theta}_{0}\right)\right] \varepsilon\left(\omega_{k}, \boldsymbol{\theta}_{0}\right), & k=l \\
0, & k \neq l
\end{array}\right.
\end{aligned}
$$


Proof: See Appendix B.

Using the above notations and properties, $\mathcal{G}$ and $\mathcal{H}$ can be derived. The first derivative of the loss function (29), evaluated at $\boldsymbol{\theta}=\boldsymbol{\theta}_{0}$, is given by

$$
J_{\boldsymbol{\theta}}^{\prime}\left(\boldsymbol{\theta}_{0}\right)=\sum_{k=n_{\omega}}^{N_{\omega}}\left[\varepsilon_{\mathcal{M}}^{*}\left(\omega_{k}\right) \mathbf{P}_{1}\left(\omega_{k}, \boldsymbol{\theta}_{0}\right) \varepsilon_{\mathcal{M}}\left(\omega_{k}\right) \quad \cdots \quad \varepsilon_{\mathcal{M}}^{*}\left(\omega_{k}\right) \mathbf{P}_{m}\left(\omega_{k}, \boldsymbol{\theta}_{0}\right) \varepsilon_{\mathcal{M}}\left(\omega_{k}\right)\right]
$$

Since $\boldsymbol{J}_{\boldsymbol{\theta}}^{\prime}\left(\boldsymbol{\theta}_{0}\right)$ is real, and using $(66)$ and $(68), \mathcal{G}_{\mathrm{DM}}$ can then be written as in

$$
\begin{aligned}
\mathcal{G}_{\mathrm{DM}} & =\mathbb{E}\left\{\left[J_{\boldsymbol{\theta}}^{\prime}\left(\boldsymbol{\theta}_{0}\right)\right]^{T} J_{\boldsymbol{\theta}}^{\prime}\left(\boldsymbol{\theta}_{0}\right)\right\}=\mathbb{E}\left\{\left[J_{\boldsymbol{\theta}}^{\prime}\left(\boldsymbol{\theta}_{0}\right)\right]^{*} J_{\boldsymbol{\theta}}^{\prime}\left(\boldsymbol{\theta}_{0}\right)\right\} \\
& =\lambda^{2} \sum_{k=n_{\omega}}^{N_{\omega}}\left[\begin{array}{cccc}
2 \varepsilon^{*} \mathbf{P}_{1}^{2} \varepsilon & \cdots & \varepsilon^{*}\left(\mathbf{P}_{1} \mathbf{P}_{m}+\mathbf{P}_{m} \mathbf{P}_{1}\right) \varepsilon \\
\vdots & \ddots & \vdots \\
\varepsilon^{*}\left(\mathbf{P}_{m} \mathbf{P}_{1}+\mathbf{P}_{1} \mathbf{P}_{m}\right) \varepsilon & \cdots & 2 \varepsilon^{*} \mathbf{P}_{m}^{2} \varepsilon
\end{array}\right] \\
& =\lambda^{2} \sum_{k=n_{\omega}}^{N_{\omega}}\left[\begin{array}{ccc}
2 \mathbf{c}^{*} \mathbf{A}_{1}^{*} \mathbf{P} \mathbf{A}_{1} \mathbf{c} & \cdots & \mathbf{c}^{*}\left(\mathbf{A}_{1}^{*} \mathbf{P} \mathbf{A}_{m}+\mathbf{A}_{m}^{*} \mathbf{P} \mathbf{A}_{1}\right) \mathbf{c} \\
\vdots & \ddots & \vdots \\
\mathbf{c}^{*}\left(\mathbf{A}_{m}^{*} \mathbf{P} \mathbf{A}_{1}+\mathbf{A}_{1}^{*} \mathbf{P} \mathbf{A}_{m}\right) \mathbf{c} & \cdots & 2 \mathbf{c}^{*} \mathbf{A}_{m}^{*} \mathbf{P} \mathbf{A}_{m} \mathbf{c}
\end{array}\right] .
\end{aligned}
$$

The arguments $\omega_{k}$ and $\boldsymbol{\theta}_{0}$ have in this equation been omitted for brevity. The second order derivative of the loss function, evaluated at $\boldsymbol{\theta}=\boldsymbol{\theta}_{0}$, is finally given by

$J_{\boldsymbol{\theta}}^{\prime \prime}\left(\boldsymbol{\theta}_{0}\right)=\sum_{k=n_{\omega}}^{N_{\omega}}\left[\begin{array}{ccc}\varepsilon_{\mathcal{M}}^{*}\left(\omega_{k}\right) \mathbf{P}_{11}\left(\omega_{k}, \boldsymbol{\theta}_{0}\right) \boldsymbol{\varepsilon}_{\mathcal{M}}\left(\omega_{k}\right) & \cdots & \boldsymbol{\varepsilon}_{\mathcal{M}}^{*}\left(\omega_{k}\right) \mathbf{P}_{1 m}\left(\omega_{k}, \boldsymbol{\theta}_{0}\right) \boldsymbol{\varepsilon}_{\mathcal{M}}\left(\omega_{k}\right) \\ \vdots & \ddots & \vdots \\ \boldsymbol{\varepsilon}_{\mathcal{M}}^{*}\left(\omega_{k}\right) \mathbf{P}_{1 m}\left(\omega_{k}, \boldsymbol{\theta}_{0}\right) \boldsymbol{\varepsilon}_{\mathcal{M}}\left(\omega_{k}\right) & \cdots & \boldsymbol{\varepsilon}_{\mathcal{M}}^{*}\left(\omega_{k}\right) \mathbf{P}_{m m}\left(\omega_{k}, \boldsymbol{\theta}_{0}\right) \boldsymbol{\varepsilon}_{\mathcal{M}}\left(\omega_{k}\right)\end{array}\right]$

and hence $\mathcal{H}_{\mathrm{DM}}$ can be expressed as in

$$
\begin{aligned}
\mathcal{H}_{\mathrm{DM}} & =\lim _{\mathrm{SNR} \rightarrow \infty} J_{\boldsymbol{\theta}}^{\prime \prime}\left(\boldsymbol{\theta}_{0}\right) \\
& =\sum_{k=n_{\omega}}^{N_{\omega}}\left[\begin{array}{ccc}
\varepsilon^{*}\left(\omega_{k}\right) \mathbf{P}_{11}\left(\omega_{k}, \boldsymbol{\theta}_{0}\right) \varepsilon\left(\omega_{k}\right) & \cdots & \varepsilon^{*}\left(\omega_{k}\right) \mathbf{P}_{1 m}\left(\omega_{k}, \boldsymbol{\theta}_{0}\right) \varepsilon\left(\omega_{k}\right) \\
\vdots & \ddots & \vdots \\
\varepsilon^{*}\left(\omega_{k}\right) \mathbf{P}_{1 m}\left(\omega_{k}, \boldsymbol{\theta}_{0}\right) \varepsilon\left(\omega_{k}\right) & \cdots & \varepsilon^{*}\left(\omega_{k}\right) \mathbf{P}_{m m}\left(\omega_{k}, \boldsymbol{\theta}_{0}\right) \varepsilon\left(\omega_{k}\right)
\end{array}\right] \\
& =\sum_{k=n_{\omega}}^{N_{\omega}}\left[\begin{array}{ccc}
2 \mathbf{c}^{*} \mathbf{A}_{1}^{*} \mathbf{P} \mathbf{A}_{1} \mathbf{c} & \cdots & \mathbf{c}^{*}\left(\mathbf{A}_{1}^{*} \mathbf{P} \mathbf{A}_{m}+\mathbf{A}_{m}^{*} \mathbf{P} \mathbf{A}_{1}\right) \mathbf{c} \\
\vdots & \ddots & \vdots \\
\mathbf{c}^{*}\left(\mathbf{A}_{m}^{*} \mathbf{P} \mathbf{A}_{1}+\mathbf{A}_{1}^{*} \mathbf{P} \mathbf{A}_{m}\right) \mathbf{c} & \cdots & 2 \mathbf{c}^{*} \mathbf{A}_{m}^{*} \mathbf{P} \mathbf{A}_{m} \mathbf{c}
\end{array}\right] \\
& =\frac{1}{\lambda^{2}} \mathcal{G}_{\mathrm{DM}} .
\end{aligned}
$$

In the third equality of (72), (67) has been used and the arguments omitted for brevity. 


\subsection{Comparison of Accuracy Between the Nonparametric Based and the Modified Data Approach}

In this section, the accuracy of the estimate achieved from the nonparametric estimate is compared to the accuracy of the modified data approach. Using (46) and (48) for the nonparametric based approach, and (70) and (72) for the modified data approach, the general expression for the covariance matrix in (33) can for each of the estimates be rewritten as

$$
\begin{aligned}
P_{\mathrm{NP}} & =\mathcal{H}_{\mathrm{NP}}^{-1} \\
P_{\mathrm{DM}} & =\lambda^{2} \mathcal{H}_{\mathrm{DM}}^{-1} .
\end{aligned}
$$

For comparing these two expressions, the following properties are needed.

Property 4: Express the vector $\Psi(\omega, \boldsymbol{\theta})$ in $(44)$ as

$$
\begin{aligned}
& \Psi(\omega, \boldsymbol{\theta})=-\left[\frac{\mathrm{d}}{\mathrm{d} \boldsymbol{\theta}} E(\omega, \boldsymbol{\theta})\right]^{T} \\
& =\left[\begin{array}{lll}
E_{1}(\omega, \boldsymbol{\theta}) & \cdots & E_{m}(\omega, \boldsymbol{\theta})
\end{array}\right]^{T} .
\end{aligned}
$$

Then $\Psi(\omega, \boldsymbol{\theta}) \Psi^{*}(\omega, \boldsymbol{\theta})$ used in the expressions for $\mathcal{G}_{\mathrm{NP}}$ and $\mathcal{H}_{\mathrm{NP}}$ in (46) and (48) is given by

$$
\Psi(\omega, \boldsymbol{\theta}) \Psi^{*}(\omega, \boldsymbol{\theta})=\left[\begin{array}{ccc}
\left|E_{1}(\omega, \boldsymbol{\theta})\right|^{2} & \cdots & E_{1}(\omega, \boldsymbol{\theta}) E_{m}^{*}(\omega, \boldsymbol{\theta}) \\
\vdots & \ddots & \vdots \\
E_{m}(\omega, \boldsymbol{\theta}) E_{1}^{*}(\omega, \boldsymbol{\theta}) & \cdots & \left|E_{m}(\omega, \boldsymbol{\theta})\right|^{2}
\end{array}\right] .
$$

Property 5: The derivative of $\mathbf{A}(\omega, \boldsymbol{\theta})$ with respect to $\theta_{i}$ is given by

$$
\mathbf{A}_{i}(\omega, \boldsymbol{\theta})=\mathbf{A}_{\mathcal{R}}(\omega, \boldsymbol{\theta}) E_{i}(\omega, \boldsymbol{\theta})
$$

where $\mathbf{A}_{\mathcal{R}}(\omega, \boldsymbol{\theta})$ is the derivative of $\mathbf{A}(\omega, \boldsymbol{\theta})$ with respect of the real part of the complex modulus.

Proof: See Appendix C.

Using Property 5 above, the following holds

$$
\begin{aligned}
\mathbf{c}^{*}\left[\mathbf{A}_{i}^{*} \mathbf{P} \mathbf{A}_{j}+\mathbf{A}_{j}^{*} \mathbf{P} \mathbf{A}_{i}\right] \mathbf{c} & =E_{i}^{*} E_{j} \underbrace{\mathbf{c}^{*} \mathbf{A}_{\mathcal{R}}^{*} \mathbf{P} \mathbf{A}_{\mathcal{R}} \mathbf{c}}_{h(\omega)}+E_{j}^{*} E_{i} \underbrace{\mathbf{c}^{*} \mathbf{A}_{\mathcal{R}}^{*} \mathbf{P} \mathbf{A}_{\mathcal{R}} \mathbf{c}}_{h(\omega)} \\
& =\frac{\lambda^{2}}{C(\omega)}\left[E_{i}^{*} E_{j}+E_{j}^{*} E_{i}\right],
\end{aligned}
$$

where (11) is used in the first equality, and (9)-(10) in the second. In (78) the arguments $\omega$ and $\boldsymbol{\theta}$ have been omitted for brevity. From (78) and Property 4, 


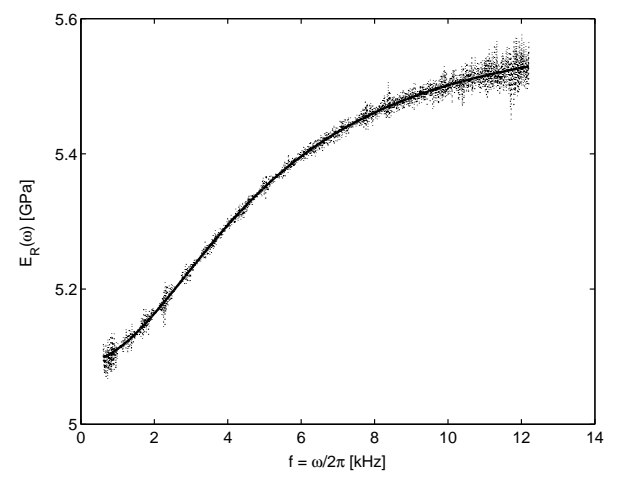

Figure 2: Real part of the estimated complex modulus for the nonparametric based approach. Nonparametric estimate (dotted) and parametric estimate (solid).

$\mathcal{H}_{\mathrm{DM}}$ in $(72)$ can be reformulated as

$$
\begin{gathered}
\mathcal{H}_{\mathrm{DM}}=\sum_{k=n_{\omega}}^{N_{\omega}} \frac{\lambda^{2}}{C\left(\omega_{k}\right)}\left[\left[\begin{array}{ccc}
\left|E_{1}\right|^{2} & \cdots & E_{1}^{*} E_{m} \\
\vdots & \ddots & \vdots \\
E_{m}^{*} E_{1} & \cdots & \left|E_{m}\right|^{2}
\end{array}\right]+\left[\begin{array}{ccc}
\left|E_{1}\right|^{2} & \cdots & E_{m}^{*} E_{1} \\
\vdots & \ddots & \vdots \\
E_{1}^{*} E_{m} & \cdots & \left|E_{m}\right|^{2}
\end{array}\right]\right] \\
=\lambda^{2} \sum_{k=n_{\omega}}^{N_{\omega}} \frac{1}{C\left(\omega_{k}\right)}\left[\Psi \Psi^{*}+\left[\Psi \Psi^{*}\right]^{T}\right]=\lambda^{2} \mathcal{H}_{\mathrm{NP}} .
\end{gathered}
$$

Using (79), the following result holds.

Result 1: Let the covariance matrix of the nonparametric based estimate and the modified data approach be given by (73) and (74), respectively. Then, by straightforward application of (79),

$$
P_{\mathrm{DM}}=\lambda^{2} \mathcal{H}_{\mathrm{DM}}^{-1}=\lambda^{2} \frac{1}{\lambda^{2}} \mathcal{H}_{\mathrm{NP}}^{-1}=P_{\mathrm{NP}}
$$

The nonparametric based approach and the modified data approach obviously result in the same theoretical accuracy of the estimates.

\section{Simulation Study}

In order to investigate the validity of the accuracy expressions given in Section 3, the parametric identifications techniques in Section 2 were considered in a Monte Carlo simulation study. The data was generated from (14), with the complex modulus given by the standard linear solid model $[6,17]$

$$
E(\omega)=\frac{E_{2}\left(E_{1}+\mathrm{i} \omega \eta\right)}{E_{1}+E_{2}+\mathrm{i} \omega \eta} .
$$


The model parameters were given by $E_{1}=56 \mathrm{GPa}, E_{2}=5.6 \mathrm{GPa}$ and $\eta=$ $2 \mathrm{MPa} \cdot \mathrm{s}$, which applies to the dynamic behavior of PMMA (plexiglass), a material with density $\rho=1183 \mathrm{~kg} / \mathrm{m}^{3}$. The length of the simulated bar was $L=2 \mathrm{~m}$, the number of data points $N=2^{15}$, and the sampling interval $T=10 \mu \mathrm{s}$. The impulse strain excitation at the right end of the bar was chosen to imitate a standard hammer excitation. The sensor locations were $\left\{x_{i}\right\}_{i=n}^{n}=\{0,0.290,0.646,1.078,1.600\} \mathrm{m}$, and Gaussian white noise with variance $\lambda^{2}=2.1 \cdot 10^{-14}$ was added to each sensor output. This procedure was repeated 100 times for different noise realizations.

Note that a free end at $x=0$ is used for the simulated data. The strains at this section are known to be zero, and can hence be used as noise-free measurements. In the simulations, however, noise was added also to the free end strains. For the nonparametric based and the modified data approach, strain measurements from all five sensors were used in the parametric identification. For the unmodified data approach, however, strain measurements from only three of the sensors were used to fit the parametric model. The fourth strain measurement at $x=0.290 \mathrm{~m}$, and the (noise-free) free end boundary condition were here used to determine the unknown amplitudes $P(\omega)$ and $N(\omega)$.

In the following, only the real parts of the estimated complex moduli are considered. The corresponding results for the imaginary parts display a similar behavior, and will therefore not be shown here. Furthermore, the nonparametric estimate of the complex modulus has for all three approaches been used as a benchmark for the parametric models.

For one noise realization, the estimate of the real part of the complex modulus for the nonparametric based approach is shown in Fig. 2. As the standard linear solid model in (81) was used to model the material behavior, as well as to generate the data for this study, it is possible to obtain a model that perfectly describes the results from the nonparametric identification. This is clearly seen in Fig. 2. The results of the other approaches are similar and therefore not shown here. Furthermore, the numerical values of the estimated parameter vectors are for all three approaches almost identical to the model parameters used to generate the data, and are therefore not reported.

In Fig. 3, the corresponding variances from the 100 Monte Carlo simulations are shown together with the variances achieved by the theoretical expressions in Section 3. There is a good agreement between the experimental and the theoretical variances, and the theoretical expressions in Section 3 are thus confirmed by the simulations study. It can also be seen that the accuracy of the nonparametric based approach is identical to the accuracy of the modified data approach. This is in agreement with the analysis in Section 3.4. These two approaches also give much higher accuracy than the unmodified data approach. This can be further improved upon by using the free end boundary condition as a known linear constraint, as is described in [7] and [9]. This is implicitly done in the unmodified data approach, when the (noise-free) free end boundary condition is used to identify the unknown amplitudes in the data model. 


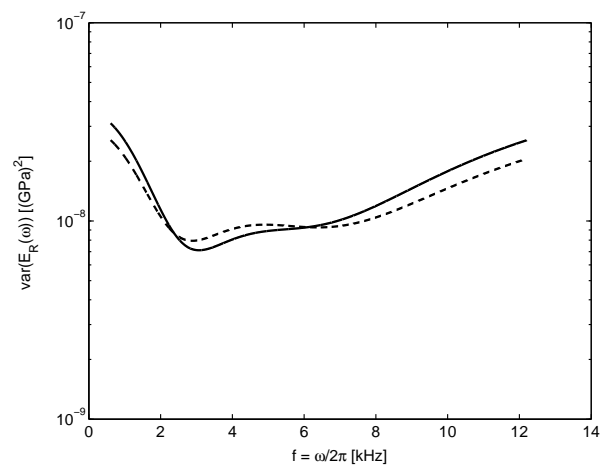

(a)

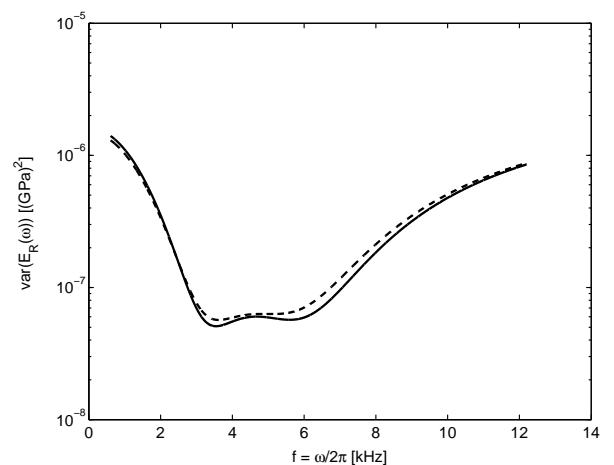

(b)

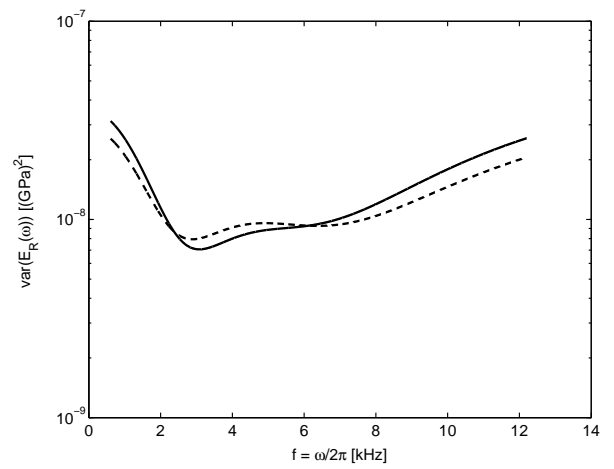

(c)

Figure 3: Variances of the real part of the estimated complex modulus. Experimental variance (solid) and theoretical variance (dashed). (a) Nonparametric based approach, (b) data approach, and (c) modified data approach.

\section{Experiments}

It is of course important that the identification techniques and accuracy expressions described in the previous sections are also applicable when real-life 


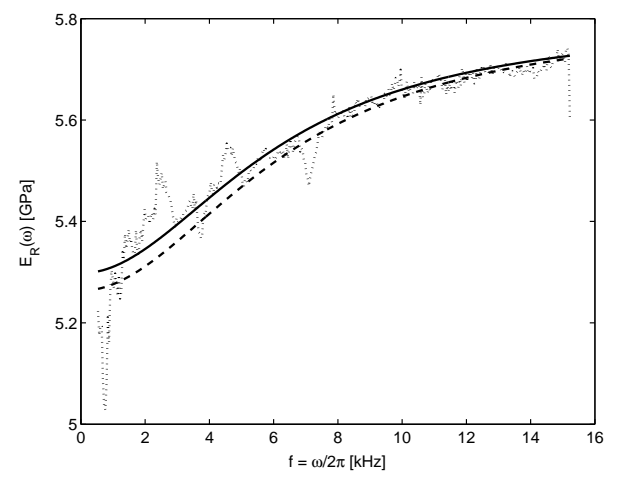

Figure 4: Real part of the estimated complex modulus. Nonparametric estimate (dotted), nonparametric based approach (solid), and the data approach (dashed). The modified data approach coincides with the nonparametric based approach.

\begin{tabular}{lccc}
\hline & $E_{1}[\mathrm{GPa}]$ & $E_{2}[\mathrm{GPa}]$ & $\eta[\mathrm{MPa} \cdot \mathrm{s}]$ \\
\hline$\hat{\boldsymbol{\theta}}_{\mathrm{NP}}$ & 62 & 5.8 & 1.8 \\
$\hat{\boldsymbol{\theta}}_{\mathrm{D}}$ & 57 & 5.8 & 1.6 \\
$\hat{\boldsymbol{\theta}}_{\mathrm{D} 2}$ & 62 & 5.8 & 1.7 \\
\hline
\end{tabular}

Table 1: Mean values of the parameter estimates from 10 independent experiments.

experimental data is used. Therefore, the three approaches were applied to data from ten independent experiments. The experiments were performed on a bar specimen made of PMMA (plexiglass), under as identical conditions as possible. The length of the bar, and the sensor locations were the same as in the simulations study in Section 4. The number of data points were $N=2^{18}$, the sampling time $T=0.1 \mu \mathrm{s}$, and the variance of the measurement noise was estimated to $\lambda^{2}=4 \cdot 10^{-11}$. Additional information on the experiments can be found in [14].

The mean values of the parameter estimates from the 10 independent experiments are shown in Table 1. In Fig. 4, the corresponding real parts of the estimated complex modulus are shown for all three approaches. Again, the standard linear solid model in (81) was used to model the material behavior. It can be seen that, using this model, the parametric estimates describe the results from the nonparametric identification in a good way. The results for the imaginary parts are similar and are therefore not shown here.

In Fig. 5, the corresponding variances are shown. In this case, the theoretical expressions underestimates the variance of the estimates in all three $\operatorname{cases}^{2}$.

\footnotetext{
${ }^{2}$ This is in agreement with the results in [11], where the nonparametric based estimate is analyzed.
} 


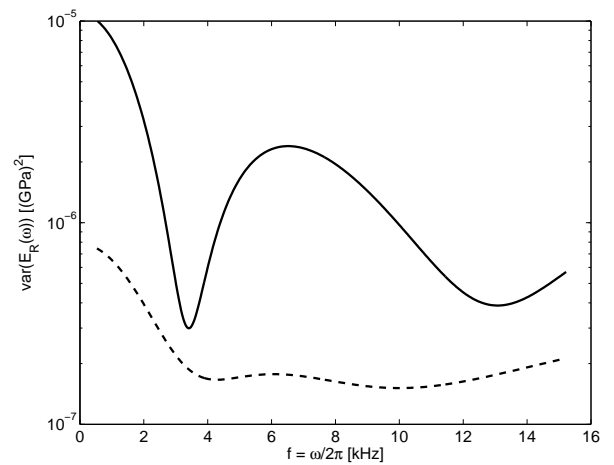

(a)

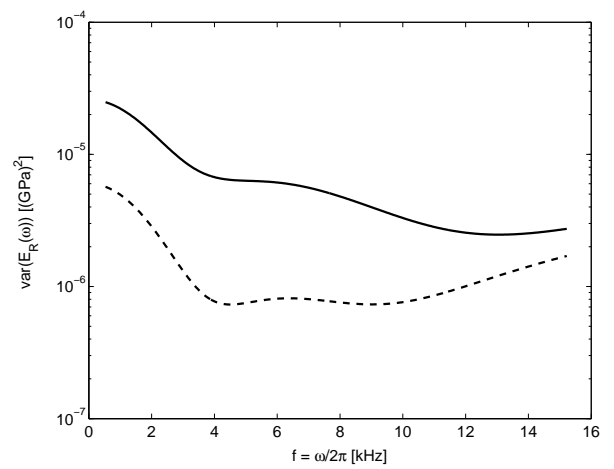

(b)

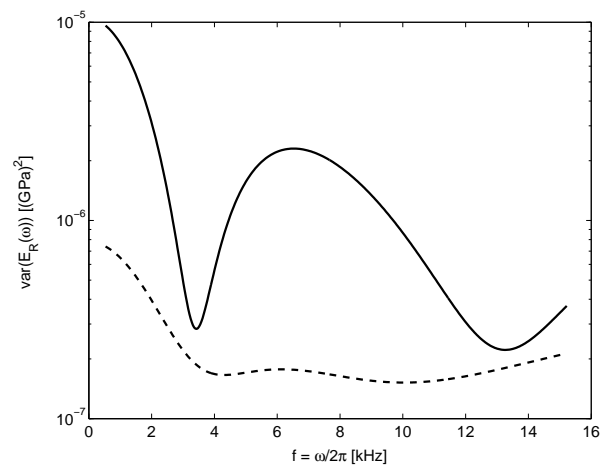

(c)

Figure 5: Variances of the real part of the estimated complex modulus. Experimental variance (solid) and theoretical variance (dashed). (a) Nonparametric based approach, (b) data approach, and (c) modified data approach.

Again, similar results are achieved for the variance of the imaginary parts. This 
underestimation may be caused by several reasons. One reason might be that the assumption on the measurement noise to be white is not valid. Another reason might be that the data model is not flexible enough to accurately describe the data. The validity of the model structure in (14) was studied in [15], and there found to be adequate for this type of experiments. In the following study, the focus will therefore be on the noise properties, since the effect of bias in the modeling of the data might be expected to be small.

\section{Accuracy Analysis - Correlated Noise}

In this section, the expression for the accuracy of the modified data approach is altered in order to cover the case of correlated measurement noise. The noise is here assumed to be zero mean, non-circular, and correlated both between frequencies and between sensors, i.e.

$$
\begin{aligned}
\mathbb{E}\left\{\mathbf{v}\left(\omega_{k}\right)\right\} & =\mathbf{0}, \forall k \\
\mathbb{E}\left\{\mathbf{v}\left(\omega_{k}\right) \mathbf{v}^{*}\left(\omega_{l}\right)\right\} & =\mathbf{R}(k, l) \\
\mathbb{E}\left\{\mathbf{v}\left(\omega_{k}\right) \mathbf{v}^{T}\left(\omega_{l}\right)\right\} & =\mathbf{Q}(k, l) .
\end{aligned}
$$

The time domain noise in this case is not a stationary process, since for a stationary process $\mathbf{R}(k, l)$ would be zero for $k \neq l$, and $\mathbf{Q}(k, l)=0$ for all $k$ and $l$, see [2]. The exact properties of the time domain noise however remains an open question, and a topic for future research. The matrices $\mathbf{R}(k, l)$ and $\mathbf{Q}(k, l)$ may be estimated from repeated experiments according to

$$
\begin{aligned}
& \mathbf{R}(k, l)=\frac{1}{M-1} \sum_{q=1}^{M}\left(\varepsilon_{\mathcal{M}}^{(q)}\left(\omega_{k}\right)-\boldsymbol{\mu}_{k}\right)\left(\varepsilon_{\mathcal{M}}^{(q)}\left(\omega_{l}\right)-\boldsymbol{\mu}_{l}\right)^{*} \\
& \mathbf{Q}(k, l)=\frac{1}{M-1} \sum_{q=1}^{M}\left(\varepsilon_{\mathcal{M}}^{(q)}\left(\omega_{k}\right)-\boldsymbol{\mu}_{k}\right)\left(\varepsilon_{\mathcal{M}}^{(q)}\left(\omega_{l}\right)-\boldsymbol{\mu}_{l}\right)^{T},
\end{aligned}
$$

where $M$ is the number of experiments and

$$
\boldsymbol{\mu}_{k}=\frac{1}{M} \sum_{q=1}^{M} \varepsilon_{\mathcal{M}}^{(q)}\left(\omega_{k}\right)
$$

To investigate how the changed noise properties influences the expression for the variance of the estimates, the following notations and properties are introduced.

Notation 3: Let

$$
\boldsymbol{\phi}_{i}(\omega, \boldsymbol{\theta})=\varepsilon^{*}(\omega, \boldsymbol{\theta}) \mathbf{P}_{i}(\omega, \boldsymbol{\theta})
$$

and

$$
\boldsymbol{\phi}(\omega, \boldsymbol{\theta})=\left[\begin{array}{lll}
\phi_{1}^{T}(\omega, \boldsymbol{\theta}) & \cdots & \boldsymbol{\phi}_{m}^{T}(\omega, \boldsymbol{\theta})
\end{array}\right]^{T} .
$$




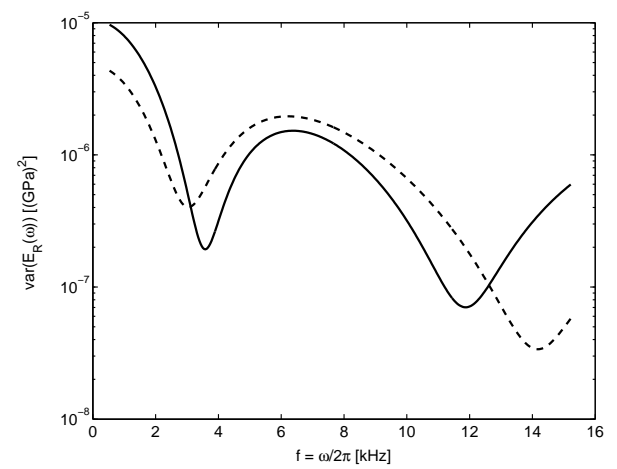

Figure 6: Variance of the real part of the complex modulus, estimated with the modified data approach. Experimental variance (solid) and theoretical variance (dashed), evaluated using correlated noise.

Property 6: Neglecting the third and fourth order moment of the noise, and using (85) and (86) above, we have that

$$
\begin{aligned}
& \mathbb{E}\left\{\varepsilon_{\mathcal{M}}^{*}\left(\omega_{k}\right) \mathbf{P}_{i}\left(\omega_{k}, \boldsymbol{\theta}_{0}\right) \varepsilon_{\mathcal{M}}\left(\omega_{k}\right) \varepsilon_{\mathcal{M}}^{*}\left(\omega_{l}\right) \mathbf{P}_{j}\left(\omega_{l}, \boldsymbol{\theta}_{0}\right) \varepsilon_{\mathcal{M}}\left(\omega_{l}\right)\right\} \\
&= \boldsymbol{\phi}_{i}\left(\omega_{k}, \boldsymbol{\theta}_{0}\right) \mathbf{R}(k, l) \boldsymbol{\phi}_{j}^{*}\left(\omega_{l}, \boldsymbol{\theta}_{0}\right) \\
&+ \boldsymbol{\phi}_{i}\left(\omega_{k}, \boldsymbol{\theta}_{0}\right) \mathbf{Q}(k, l) \boldsymbol{\phi}_{j}^{T}\left(\omega_{l}, \boldsymbol{\theta}_{0}\right) \\
&+\operatorname{conj}\{ \boldsymbol{\phi}_{i}\left(\omega_{k}, \boldsymbol{\theta}_{0}\right) \mathbf{R}(k, l) \boldsymbol{\phi}_{j}^{*}\left(\omega_{l}, \boldsymbol{\theta}_{0}\right) \\
&\left.+\boldsymbol{\phi}_{i}\left(\omega_{k}, \boldsymbol{\theta}_{0}\right) \mathbf{Q}(k, l) \boldsymbol{\phi}_{j}^{T}\left(\omega_{l}, \boldsymbol{\theta}_{0}\right)\right\}
\end{aligned}
$$

Proof: See Appendix D.

Using (69), Notation 3, and Property 6 above, $\mathcal{G}_{\mathrm{DM}}$ can be reevaluated as

$$
\begin{array}{r}
\mathcal{G}_{\mathrm{DM}^{\prime}}=\mathbb{E}\left\{\left[J_{\boldsymbol{\theta}}^{\prime}\left(\boldsymbol{\theta}_{0}\right)\right]^{T} J_{\boldsymbol{\theta}}^{\prime}\left(\boldsymbol{\theta}_{0}\right)\right\}=\mathbb{E}\left\{\left[J_{\boldsymbol{\theta}}^{\prime}\left(\boldsymbol{\theta}_{0}\right)\right]^{*} J_{\boldsymbol{\theta}}^{\prime}\left(\boldsymbol{\theta}_{0}\right)\right\} \\
=\lambda^{2} \sum_{k=n_{\omega}}^{N_{\omega}} \sum_{l=n_{\omega}}^{N_{\omega}}\left[\phi\left(\omega_{k}, \boldsymbol{\theta}_{0}\right) \mathbf{R}(k, l) \phi^{*}\left(\omega_{l}, \boldsymbol{\theta}_{0}\right)\right. \\
+\phi\left(\omega_{k}, \boldsymbol{\theta}_{0}\right) \mathbf{Q}(k, l) \phi^{T}\left(\omega_{l}, \boldsymbol{\theta}_{0}\right) \\
+\operatorname{conj}\left\{\phi\left(\omega_{k}, \boldsymbol{\theta}_{0}\right) \mathbf{R}(k, l) \phi^{*}\left(\omega_{l}, \boldsymbol{\theta}_{0}\right)\right. \\
\left.\left.+\phi\left(\omega_{k}, \boldsymbol{\theta}_{0}\right) \mathbf{Q}(k, l) \phi^{T}\left(\omega_{l}, \boldsymbol{\theta}_{0}\right)\right\}\right],
\end{array}
$$

The matrix $\mathcal{H}_{\mathrm{DM}}$ is left unchanged by the change in noise properties.

In Fig. 6, the theoretical and experimental variances of the real part of the estimated complex modulus are shown. Here, only five experiments were used to estimate the experimental variance. The remaining five experiments were used to estimate the matrices $\mathbf{R}(k, l)$ and $\mathbf{Q}(k, l)$ according to (83). It can be seen that the theoretical and the experimental variances are now comparable 
in magnitude. For this application, the correlated noise assumption seems to be more accurate than assuming the measurement noise to be white. It is however important to note that the white noise assumption is adequate in the nonparametric case, where each frequency point is considered separately. In the parametric case considered here, the effect at a large number of frequency points is accumulated, and the deviations from the white noise assumption hence become more noticeable.

\section{Conclusions}

Three different approaches to parametric identification of the complex modulus of a viscoelastic material was considered in this report. The first procedure employs a nonparametric estimate to fit the parametric model, while the other two fits the model directly from the data. All three methods were found to give good estimates of the complex modulus. Theoretical expressions for the accuracy of the estimates were also derived, assuming that the measurement noise was temporally and spatially white. The validity of these expressions was investigated both with simulated and with experimental data. In the simulated data study, the theoretical variances were in very good agreement with the experimental variances of the estimates. In the experimental data case, the theoretical values however severely underestimated the variance in all three cases. For one of the identification procedures, the theoretical accuracy expression was therefore modified to cover an arbitrary noise model. It was found that the colored noise assumption in this case gave much better agreement between the theoretical and the experimental variance.

\section{Acknowledgment}

The authors would like to thank Dr. Saed Mousavi for providing the data used in this study. This research was partially supported by the Swedish Research Council, contract 621-2007-6364.

\section{References}

[1] R. H. Blanc. Transient wave propagation methods for determining the viscoelastic properties of solids. Journal of Applied Mechanics, 60:763-768, 1993.

[2] D. R. Brillinger. Time Series: Data Analysis and Theory. Holden-Day, Inc, San Francisco, CA, USA, 1981.

[3] G. H. Golub and C. F. Van Loan. Matrix Computations, 2nd ed. John Hopkins University Press, Baltimore, MD, USA, 1989. 
[4] L. Hillström, M. Mossberg, and B. Lundberg. Identification of complex modulus from measured strains on an axially impacted bar using least squares. Journal of Sound and Vibration, 230(3):689-707, February 2000.

[5] A. J. Hull. An inverse method to measure the axial modulus of composite materials under tension. Journal of Sound and Vibration, 195:545-551, 1996.

[6] R. S. Lakes. Viscoelastic Solids. CRC Press, 1999.

[7] K. Mahata and H. Garnier. Direct identification of continuous-time errorsin-variables models. In 14th IFAC World Congress, Prague, Czech Republic, July 3-8 2005.

[8] K. Mahata, S. Mousavi, T. Söderström, M. Mossberg, U. Valdek, and L. Hillström. On the use of flexural wave propagation experiments for identification of complex modulus. IEEE Transactions on Control Systems Technology, 11(6):863-874, November 2003.

[9] K. Mahata and T. Söderström. Improved estimation performance using known linear constraints. Automatica, 40(8):1307-1318, August 2004.

[10] M. Mossberg, L. Hillström, and L. Abrahamsson. Parametric identification of viscoelastic materials from time and frequency domain data. Inverse Problems in Engineering, 9(6):645-670, December 2001.

[11] M. Mossberg, L. Hillström, and T. Söderström. Identification of viscoelastic materials. In Proc. SYSID 2000, 12th IFAC Symposium on System Identification, Santa Barbara, California, June 21-23 2000.

[12] R. Pintelon, P. Guillaume, S. Vanlanduit, K. De Belder, and Y. Rolain. Identification of Young's modulus from broadband modal analysis experiments. Mechanical Systems and Signal Processing, 18(4):699-726, July 2004.

[13] R. Pintelon and J. Schoukens. System Identification. A Frequency Domain Approach. IEEE Press, New York, NY, USA, 2001.

[14] A. Rensfelt, S. Mousavi, M. Mossberg, and T. Söderström. Optimal sensor locations for nonparametric identification of viscoelastic materials. Automatica, 44(1):28-38, January 2008.

[15] A. Rensfelt and T. Söderström. Structure testing of wave propagation models used in identification of viscoelastic materials. Automatica, 46(4):728734, April 2010.

[16] Y. Sogabe and M. Tsuzuki. Identification of the dynamic properties of linear viscoelastic materials by wave propagating testings. Bulletin of the Japan Society of Mechanical Engineers, 29:2410-2417, 1986.

[17] C. Zener. Elasticity and Anelasticity of Metals. University of Chicago Press, IL, USA, 1948. 


\section{A Proof of Property 2}

For the first and second derivative of the projection operator $\mathbf{P}(\omega, \boldsymbol{\theta})$ in (12) we have the following results

$$
\begin{aligned}
\mathbf{P}_{i}(\omega, \boldsymbol{\theta})= & -\mathbf{P}(\omega, \boldsymbol{\theta}) \mathbf{A}_{i}(\omega, \boldsymbol{\theta}) \mathbf{A}^{\dagger}(\omega, \boldsymbol{\theta})-\left[\mathbf{P}(\omega, \boldsymbol{\theta}) \mathbf{A}_{i}(\omega, \boldsymbol{\theta}) \mathbf{A}^{\dagger}(\omega, \boldsymbol{\theta})\right]^{*} \\
\mathbf{P}_{i j}(\omega, \boldsymbol{\theta})= & -\mathbf{P}_{j}(\omega, \boldsymbol{\theta}) \mathbf{A}_{i}(\omega, \boldsymbol{\theta}) \mathbf{A}^{\dagger}(\omega, \boldsymbol{\theta})-\mathbf{P}(\omega, \boldsymbol{\theta}) \mathbf{A}_{i j}(\omega, \boldsymbol{\theta}) \mathbf{A}^{\dagger}(\omega, \boldsymbol{\theta}) \\
& -\mathbf{P}(\omega, \boldsymbol{\theta}) \mathbf{A}_{i}(\omega, \boldsymbol{\theta})\left(\mathbf{A}^{\dagger}(\omega, \boldsymbol{\theta})\right)_{j}-\left[\mathbf{P}_{j}(\omega, \boldsymbol{\theta}) \mathbf{A}_{i}(\omega, \boldsymbol{\theta}) \mathbf{A}^{\dagger}(\omega, \boldsymbol{\theta})\right]^{*} \\
& -\left[\mathbf{P}(\omega, \boldsymbol{\theta}) \mathbf{A}_{i j}(\omega, \boldsymbol{\theta}) \mathbf{A}^{\dagger}(\omega, \boldsymbol{\theta})\right]^{*}-\left[\mathbf{P}(\omega, \boldsymbol{\theta}) \mathbf{A}_{i}(\omega, \boldsymbol{\theta})\left(\mathbf{A}^{\dagger}(\omega, \boldsymbol{\theta})\right)_{j}\right]^{*} .
\end{aligned}
$$

For proof, the reader is referred to [3]. Since

$$
\mathbf{P}(\omega, \boldsymbol{\theta}) \mathbf{A}(\omega, \boldsymbol{\theta})=\mathbf{P}^{*}(\omega, \boldsymbol{\theta}) \mathbf{A}(\omega, \boldsymbol{\theta})=\mathbf{0},
$$

and using (26), we get

$$
\begin{aligned}
\varepsilon^{*} \mathbf{P}_{i} \mathbf{P}_{j} \varepsilon & =\varepsilon^{*}\left[\mathbf{P A}_{i} \mathbf{A}^{\dagger}+\left[\mathbf{P A}_{i} \mathbf{A}^{\dagger}\right]^{*}\right]\left[\mathbf{P A}_{j} \mathbf{A}^{\dagger}+\left[\mathbf{P A}_{j} \mathbf{A}^{\dagger}\right]^{*}\right] \varepsilon \\
& =\mathbf{c}^{*} \mathbf{A}^{*}\left[\mathbf{P} \mathbf{A}_{i} \mathbf{A}^{\dagger}+\mathbf{A}^{\dagger *} \mathbf{A}_{i}^{*} \mathbf{P}^{*}\right]\left[\mathbf{P} \mathbf{A}_{j} \mathbf{A}^{\dagger}+\mathbf{A}^{\dagger *} \mathbf{A}_{j}^{*} \mathbf{P}^{*}\right] \mathbf{A} \mathbf{c} \\
& =\mathbf{c}^{*} \underbrace{\mathbf{A}^{*} \mathbf{A}^{\dagger *}}_{=\mathbf{I}} \mathbf{A}_{i}^{*} \mathbf{P}^{*} \mathbf{P} \mathbf{A}_{j} \underbrace{\mathbf{A}^{\dagger} \mathbf{A}}_{=\mathbf{I}} \mathbf{c}=\mathbf{c}^{*} \mathbf{A}_{i}^{*} \underbrace{\mathbf{P}^{*} \mathbf{P}}_{=\mathbf{P}} \mathbf{A}_{j} \mathbf{c} \\
& =\mathbf{c}^{*} \mathbf{A}_{i}^{*} \mathbf{P} \mathbf{A}_{j} \mathbf{c},
\end{aligned}
$$

which proves (66). In (92), the arguments $\omega$ and $\boldsymbol{\theta}$ are omitted for brevity. Similarly, using (90) we have that

$$
\begin{aligned}
\varepsilon^{*} \mathbf{P}_{i j} \varepsilon= & -\varepsilon^{*}\left[\mathbf{P}_{j} \mathbf{A}_{i} \mathbf{A}^{\dagger}+\mathbf{P} \mathbf{A}_{i j} \mathbf{A}^{\dagger}+\mathbf{P} \mathbf{A}_{i}\left(\mathbf{A}^{\dagger}\right)_{j}\right. \\
& \left.+\left[\mathbf{P}_{j} \mathbf{A}_{i} \mathbf{A}^{\dagger}\right]^{*}+\left[\mathbf{P} \mathbf{A}_{i j} \mathbf{A}^{\dagger}\right]^{*}+\left[\mathbf{P A}_{i}\left(\mathbf{A}^{\dagger}\right)_{j}\right]^{*}\right] \varepsilon \\
= & -\mathbf{c}^{*} \mathbf{A}^{*}\left[\mathbf{P}_{j} \mathbf{A}_{i} \mathbf{A}^{\dagger}+\mathbf{P} \mathbf{A}_{i j} \mathbf{A}^{\dagger}+\mathbf{P} \mathbf{A}_{i}\left(\mathbf{A}^{\dagger}\right)_{j}\right. \\
& \left.+\mathbf{A}^{\dagger *} \mathbf{A}_{i}^{*} \mathbf{P}_{j}^{*}+\mathbf{A}^{\dagger *} \mathbf{A}_{i j}^{*} \mathbf{P}^{*}+\left(\mathbf{A}^{\dagger}\right)_{j}^{*} \mathbf{A}_{i}^{*} \mathbf{P}^{*}\right] \mathbf{A} \mathbf{c} \\
= & -\mathbf{c}^{*}[\mathbf{A}^{*} \mathbf{P}_{j} \mathbf{A}_{i} \underbrace{\mathbf{A}^{\dagger} \mathbf{A}}_{=\mathbf{I}}+\underbrace{\mathbf{A}^{*} \mathbf{A}^{\dagger *}}_{=\mathbf{I}} \mathbf{A}_{i}^{*} \mathbf{P}_{j}^{*} \mathbf{A}] \mathbf{c} \\
= & -\mathbf{c}^{*}\left[\mathbf{A}^{*} \mathbf{P}_{j} \mathbf{A}_{i}+\mathbf{A}_{i}^{*} \mathbf{P}_{j}^{*} \mathbf{A}\right] \mathbf{c} \\
= & -\mathbf{c}^{*} \mathbf{A}^{*}\left[-\mathbf{P} \mathbf{A}_{j} \mathbf{A}^{\dagger}-\left[\mathbf{P} \mathbf{A}_{j} \mathbf{A}^{\dagger}\right]^{*}\right] \mathbf{A}_{i} \mathbf{c} \\
& -\mathbf{c}^{*} \mathbf{A}_{i}^{*}\left[-\left[\mathbf{P} \mathbf{A}_{j} \mathbf{A}^{\dagger}\right]^{*}-\mathbf{P} \mathbf{A}_{j} \mathbf{A}^{\dagger}\right] \mathbf{A} \mathbf{c} \\
= & \mathbf{c}^{*} \underbrace{\mathbf{A}^{*} \mathbf{A}^{\dagger *}}_{=\mathbf{I}} \mathbf{A}_{j}^{*} \mathbf{P}^{*} \mathbf{A}_{i} \mathbf{c}+\mathbf{c}^{*} \mathbf{A}_{i}^{*} \mathbf{P} \mathbf{A}_{j} \underbrace{\mathbf{A}^{\dagger} \mathbf{A} \mathbf{c}}_{=\mathbf{I}} \\
= & \mathbf{c}^{*} \mathbf{A}_{j}^{*} \mathbf{P}^{*} \mathbf{A}_{i} \mathbf{c}+\mathbf{c}^{*} \mathbf{A}_{i}^{*} \mathbf{P} \mathbf{A}_{j} \mathbf{c},
\end{aligned}
$$

which proves (67). 


\section{B Proof of Property 3}

For this proof, the following result and property are needed.

Result 2: Let $\mathbf{v}(\omega)$ be a complex Gaussian random vector with properties

$$
\begin{aligned}
\mathbb{E}\left\{\mathbf{v}\left(\omega_{k}\right)\right\} & =\mathbf{0}, \\
\mathbb{E}\left\{\mathbf{v}\left(\omega_{k}\right) \mathbf{v}^{T}\left(\omega_{l}\right)\right\} & =\mathbf{0}, \forall k, l \text { s.t. } 0<k, l<\frac{N}{2}, \\
\mathbb{E}\left\{\mathbf{v}\left(\omega_{k}\right) \mathbf{v}^{*}\left(\omega_{l}\right)\right\} & =\lambda^{2} \mathbf{I}, \forall k, l \text { s.t. } 0<k, l<\frac{N}{2} .
\end{aligned}
$$

Let $\mathbf{x}$ be a complex valued deterministic vector of the same dimension as $\mathbf{v}(\omega)$.

Let $\mathbf{R}$ and $\mathbf{S}$ be matrices of compatible dimensions such that

$$
\mathbf{x}^{*} \mathbf{R} \mathbf{x}=\mathbf{x}^{*} \mathbf{S x}=0 .
$$

Then

$$
\begin{gathered}
\mathbb{E}\left\{\left[\mathbf{x}+\mathbf{v}\left(\omega_{k}\right)\right]^{*} \mathbf{R}\left[\mathbf{x}+\mathbf{v}\left(\omega_{k}\right)\right]\left[\mathbf{x}+\mathbf{v}\left(\omega_{k}\right)\right]^{*} \mathbf{S}\left[\mathbf{x}+\mathbf{v}\left(\omega_{k}\right)\right]\right\} \\
=\lambda^{2} \mathbf{x}^{*}[\mathbf{R S}+\mathbf{S R}] \mathbf{x}+\lambda^{4}[\operatorname{tr}\{\mathbf{S}\} \operatorname{tr}\{\mathbf{R}\}+\operatorname{tr}\{\mathbf{S R}\}]
\end{gathered}
$$

Proof: See [8].

Property 7: The following holds

$$
\operatorname{tr}\left\{\mathbf{P}_{i}\left(\omega, \boldsymbol{\theta}_{0}\right)\right\}=0 .
$$

Proof: See [10].

The measured data vector $\varepsilon_{\mathcal{M}}(\omega)$ used in Property 3 is given by

$$
\varepsilon_{\mathcal{M}}(\omega)=\varepsilon\left(\omega, \boldsymbol{\theta}_{0}\right)+\mathbf{v}(\omega),
$$

where $\mathbf{v}(\omega)$ is a noise vector with properties as in (94)-(96). Using (26), (89) and (91) we have that

$$
\begin{aligned}
\varepsilon^{*}\left(\omega, \boldsymbol{\theta}_{0}\right) \mathbf{P}_{i}\left(\omega, \boldsymbol{\theta}_{0}\right) \varepsilon\left(\omega, \boldsymbol{\theta}_{0}\right) & =\varepsilon^{*}\left[\mathbf{P} \mathbf{A}_{i} \mathbf{A}^{\dagger}+\left[\mathbf{P} \mathbf{A}_{i} \mathbf{A}^{\dagger}\right]^{*}\right] \varepsilon \\
& =\mathbf{c}^{*} \mathbf{A}^{*}\left[\mathbf{P} \mathbf{A}_{i} \mathbf{A}^{\dagger}+\mathbf{A}^{\dagger^{*}} \mathbf{A}_{i}^{*} \mathbf{P}^{*}\right] \mathbf{A} \mathbf{c}=0 .
\end{aligned}
$$

Furthermore, using (94), (96), (101) and Property 7 we have that

$$
\begin{aligned}
\mathbb{E}\left\{\varepsilon_{\mathcal{M}}^{*}\left(\omega_{k}\right) \mathbf{P}_{i}\left(\omega_{k}, \boldsymbol{\theta}_{0}\right) \varepsilon_{\mathcal{M}}\left(\omega_{k}\right)\right\} & =\mathbb{E}\left\{\mathbf{v}^{*}\left(\omega_{k}\right) \mathbf{P}_{i}\left(\omega_{k}, \boldsymbol{\theta}_{0}\right) \mathbf{v}\left(\omega_{k}\right)\right\} \\
& =\operatorname{tr}\left\{\mathbf{P}_{i}\left(\omega_{k}, \boldsymbol{\theta}_{0}\right) \mathbb{E}\left\{\mathbf{v}^{*}\left(\omega_{k}\right) \mathbf{v}\left(\omega_{k}\right)\right\}\right\} \\
& =\lambda^{2} \operatorname{tr}\left\{\mathbf{P}_{i}\left(\omega_{k}, \boldsymbol{\theta}_{0}\right)\right\}=0 .
\end{aligned}
$$

The following result can now be formulated. 
Result 3: Let $\varepsilon_{\mathcal{M}}(\omega)$ be given by (100) with noise properties as in (94)-(96). Then

$$
\begin{aligned}
\mathbb{E} & \left\{\varepsilon_{\mathcal{M}}^{*}\left(\omega_{k}\right) \mathbf{P}_{i}\left(\omega_{k}, \boldsymbol{\theta}_{0}\right) \varepsilon_{\mathcal{M}}\left(\omega_{k}\right) \varepsilon_{\mathcal{M}}^{*}\left(\omega_{l}\right) \mathbf{P}_{j}\left(\omega_{l}, \boldsymbol{\theta}_{0}\right) \varepsilon_{\mathcal{M}}\left(\omega_{l}\right)\right\} \\
& =\left\{\begin{array}{cc}
\lambda^{2} \varepsilon^{*}\left[\mathbf{P}_{i} \mathbf{P}_{j}+\mathbf{P}_{j} \mathbf{P}_{i}\right] \varepsilon \\
+\lambda^{4}\left[\operatorname{tr}\left\{\mathbf{P}_{i}\right\} \operatorname{tr}\left\{\mathbf{P}_{j}\right\}+\operatorname{tr}\left\{\mathbf{P}_{j}\right\} \operatorname{tr}\left\{\mathbf{P}_{i}\right\}\right], & k=l \\
0, & k \neq l
\end{array}\right.
\end{aligned}
$$

Proof: For $k=l$, the result follows from direct application of (100), (101), and Result 2 above. For $k \neq l$ we have that uncorrelated random vectors are independent, and hence

$$
\begin{aligned}
\mathbb{E}\left\{\varepsilon_{\mathcal{M}}^{*}\left(\omega_{k}\right) \mathbf{P}_{i}\left(\omega_{k}, \boldsymbol{\theta}_{0}\right) \varepsilon_{\mathcal{M}}\left(\omega_{k}\right) \varepsilon_{\mathcal{M}}^{*}\left(\omega_{l}\right) \mathbf{P}_{j}\left(\omega_{l}, \boldsymbol{\theta}_{0}\right) \varepsilon_{\mathcal{M}}\left(\omega_{l}\right)\right\} \\
=\mathbb{E}\left\{\varepsilon_{\mathcal{M}}^{*}\left(\omega_{k}\right) \mathbf{P}_{i}\left(\omega_{k}, \boldsymbol{\theta}_{0}\right) \varepsilon_{\mathcal{M}}\left(\omega_{k}\right)\right\} \\
\times \mathbb{E}\left\{\varepsilon_{\mathcal{M}}^{*}\left(\omega_{l}\right) \mathbf{P}_{j}\left(\omega_{l}, \boldsymbol{\theta}_{0}\right) \varepsilon_{\mathcal{M}}\left(\omega_{l}\right)\right\}=0
\end{aligned}
$$

by the use of (102).

Neglecting the fourth order moment of the noise in (103), the expression in (68) is received.

\section{Proof of Property 5}

Differentiating the elements in the second column of $\mathbf{A}(\omega, \boldsymbol{\theta})$ with respect to the real part of the complex modulus gives

$$
\begin{aligned}
\left(\mathrm{e}^{\gamma(\omega, \boldsymbol{\theta}) x_{i}}\right)_{\mathcal{R}} & =\frac{\partial \mathrm{e}^{\gamma(\omega, \boldsymbol{\theta}) x_{i}}}{\partial E_{\mathcal{R}}(\omega, \boldsymbol{\theta})}=x_{i} \mathrm{e}^{\gamma(\omega, \boldsymbol{\theta}) x_{i}} \frac{\partial \gamma(\omega, \boldsymbol{\theta})}{\partial E_{\mathcal{R}}(\omega, \boldsymbol{\theta})} \\
& =x_{i} \mathrm{e}^{\gamma(\omega, \boldsymbol{\theta}) x_{i}} \frac{\partial \gamma(\omega, \boldsymbol{\theta})}{\partial E(\omega, \boldsymbol{\theta})} \underbrace{\frac{\partial E(\omega, \boldsymbol{\theta})}{\partial E_{\mathcal{R}}(\omega, \boldsymbol{\theta})}}_{=1} \\
& =x_{i} \mathrm{e}^{\gamma(\omega, \boldsymbol{\theta}) x_{i}} \frac{\partial \gamma(\omega, \boldsymbol{\theta})}{\partial E(\omega, \boldsymbol{\theta})}
\end{aligned}
$$

Analogous results are obtained for the elements of the first column of $\mathbf{A}(\omega, \boldsymbol{\theta})$. Similarly, differentiating the elements of $\mathbf{A}(\omega, \boldsymbol{\theta})$ with respect to $\theta_{i}$ gives

$$
\begin{aligned}
\left(\mathrm{e}^{\gamma(\omega, \boldsymbol{\theta}) x_{i}}\right)_{i} & =\frac{\partial \mathrm{e}^{\gamma(\omega, \boldsymbol{\theta}) x_{i}}}{\partial \theta_{i}}=x_{i} \mathrm{e}^{\gamma(\omega, \boldsymbol{\theta}) x_{i}} \frac{\partial \gamma(\omega, \boldsymbol{\theta})}{\partial \theta_{i}} \\
& =x_{i} \mathrm{e}^{\gamma(\omega, \boldsymbol{\theta}) x_{i}} \frac{\partial \gamma(\omega, \boldsymbol{\theta})}{\partial E(\omega, \boldsymbol{\theta})} \frac{\partial E(\omega, \boldsymbol{\theta})}{\partial \theta_{i}} \\
& =\left(\mathrm{e}^{\gamma(\omega, \boldsymbol{\theta}) x_{i}}\right)_{\mathcal{R}} E_{i}(\omega, \boldsymbol{\theta}),
\end{aligned}
$$

with analogous results for the elements of the first column. Stacking the elements into matrices we hence get

$$
\mathbf{A}_{i}(\omega, \boldsymbol{\theta})=\mathbf{A}_{\mathcal{R}}(\omega, \boldsymbol{\theta}) E_{i}(\omega, \boldsymbol{\theta}) .
$$




\section{Proof of Property 6}

Using (85), (101), and neglecting the third and fourth order moments of the noise, we have that

$$
\begin{aligned}
& \mathbb{E}\left\{\varepsilon_{\mathcal{M}}^{*}\left(\omega_{k}\right) \mathbf{P}_{i}\left(\omega_{k}\right) \varepsilon_{\mathcal{M}}\left(\omega_{k}\right) \varepsilon_{\mathcal{M}}^{*}\left(\omega_{l}\right) \mathbf{P}_{j}\left(\omega_{l}\right) \varepsilon_{\mathcal{M}}\left(\omega_{l}\right)\right\} \\
&=\mathbb{E}\left\{\left[\varepsilon\left(\omega_{k}\right)+\mathbf{v}\left(\omega_{k}\right)\right]^{*} \mathbf{P}_{i}\left(\omega_{k}\right)\left[\varepsilon\left(\omega_{k}\right)+\mathbf{v}\left(\omega_{k}\right)\right]\right. \\
&\left.\quad \times\left[\varepsilon\left(\omega_{l}\right)+\mathbf{v}\left(\omega_{l}\right)\right]^{*} \mathbf{P}_{j}\left(\omega_{l}\right)\left[\varepsilon\left(\omega_{l}\right)+\mathbf{v}\left(\omega_{l}\right)\right]\right\} \\
&=\mathbb{E}\left\{\boldsymbol{\phi}_{i}\left(\omega_{k}\right) \mathbf{v}\left(\omega_{k}\right) \boldsymbol{\phi}_{j}\left(\omega_{l}\right) \mathbf{v}\left(\omega_{l}\right)\right\}+\phi_{i}\left(\omega_{k}\right) \mathbb{E}\left\{\mathbf{v}\left(\omega_{k}\right) \mathbf{v}^{*}\left(\omega_{l}\right)\right\} \boldsymbol{\phi}_{j}^{*}\left(\omega_{l}\right) \\
&+\mathbb{E}\left\{\mathbf{v}^{*}\left(\omega_{k}\right) \boldsymbol{\phi}_{i}^{*}\left(\omega_{k}\right) \boldsymbol{\phi}_{j}\left(\omega_{l}\right) \mathbf{v}\left(\omega_{l}\right)\right\}+\mathbb{E}\left\{\mathbf{v}^{*}\left(\omega_{k}\right) \boldsymbol{\phi}_{i}^{*}\left(\omega_{k}\right) \mathbf{v}^{*}\left(\omega_{l}\right) \boldsymbol{\phi}_{j}^{*}\left(\omega_{l}\right)\right\} \\
&= \phi_{i}\left(\omega_{k}\right) \mathbb{E}\left\{\mathbf{v}\left(\omega_{k}\right) \mathbf{v}^{T}\left(\omega_{l}\right)\right\} \boldsymbol{\phi}_{j}^{T}\left(\omega_{l}\right)+\boldsymbol{\phi}_{i}\left(\omega_{k}\right) \mathbb{E}\left\{\mathbf{v}\left(\omega_{k}\right) \mathbf{v}^{*}\left(\omega_{l}\right)\right\} \boldsymbol{\phi}_{j}^{*}\left(\omega_{l}\right) \\
&+\boldsymbol{\phi}_{j}\left(\omega_{l}\right) \mathbb{E}\left\{\mathbf{v}\left(\omega_{l}\right) \mathbf{v}^{*}\left(\omega_{k}\right)\right\} \boldsymbol{\phi}_{i}^{*}\left(\omega_{k}\right)+\left[\boldsymbol{\phi}_{i}^{*}\left(\omega_{k}\right)\right]^{T} \mathbb{E}\left\{\left[\mathbf{v}^{T}\left(\omega_{k}\right)\right]^{*} \mathbf{v}^{*}\left(\omega_{l}\right)\right\} \boldsymbol{\phi}_{j}^{*}\left(\omega_{l}\right) \\
&= \phi_{i}\left(\omega_{k}\right) \mathbf{Q}(k, l) \boldsymbol{\phi}_{j}^{T}\left(\omega_{l}\right)+\boldsymbol{\phi}_{i}\left(\omega_{k}\right) \mathbf{R}(k, l) \boldsymbol{\phi}_{j}^{*}\left(\omega_{l}\right) \\
&+\boldsymbol{\phi}_{j}\left(\omega_{l}\right) \mathbf{R}(l, k) \boldsymbol{\phi}_{i}^{*}\left(\omega_{k}\right)+\left[\boldsymbol{\phi}_{i}^{*}\left(\omega_{k}\right)\right]^{T} \mathbf{Q}^{*}(l, k) \boldsymbol{\phi}_{j}^{*}\left(\omega_{l}\right) \\
&= \boldsymbol{\phi}_{i}\left(\omega_{k}\right) \mathbf{R}(k, l) \boldsymbol{\phi}_{j}^{*}\left(\omega_{l}\right)+\boldsymbol{\phi}_{i}\left(\omega_{k}\right) \mathbf{Q}(k, l) \boldsymbol{\phi}_{j}^{T}\left(\omega_{l}\right) \\
&+\left[\boldsymbol{\phi}_{i}\left(\omega_{k}\right) \mathbf{R}(k, l) \boldsymbol{\phi}_{j}\left(\omega_{l}\right)\right]^{*}+\left[\boldsymbol{\phi}_{i}\left(\omega_{k}\right) \mathbf{Q}(k, l) \boldsymbol{\phi}_{j}^{T}\left(\omega_{l}\right)\right]^{*} \\
&= \boldsymbol{\phi}_{i}\left(\omega_{k}\right) \mathbf{R}(k, l) \boldsymbol{\phi}_{j}^{*}\left(\omega_{l}\right)+\boldsymbol{\phi}_{i}\left(\omega_{k}\right) \mathbf{Q}(k, l) \boldsymbol{\phi}_{j}^{T}\left(\omega_{l}\right) \\
&+\operatorname{conj}\left\{\boldsymbol{\phi}_{i}\left(\omega_{k}\right) \mathbf{R}(k, l) \boldsymbol{\phi}_{j}\left(\omega_{l}\right)+\boldsymbol{\phi}_{i}\left(\omega_{k}\right) \mathbf{Q}(k, l) \boldsymbol{\phi}_{j}^{T}\left(\omega_{l}\right)\right\} .
\end{aligned}
$$

Above, we have used that $\boldsymbol{\phi}_{i}(\omega) \mathbf{v}(\omega)$ and each term of the sum are scalar, and hence equal to its transpose. We have also used that $\mathbf{R}(l, k)=\mathbf{R}^{*}(k, l)$ and $\mathbf{Q}(l, k)=\mathbf{Q}^{T}(k, l)$. The argument $\boldsymbol{\theta}_{0}$ has here been omitted for brevity. 